\title{
Homocoupling of Iodoarenes and Bromoalkanes using Photoredox Gold Catalysis: A Light Enabled Au(III) Reductive Elimination
}

\author{
Huy Tran, Terry McCallum, Mathieu Morin and Louis Barriault* \\ Centre for Catalysis, Research and Innovation, Department of Chemistry and Biomolecular Sciences, \\ University of Ottawa, Ottawa, Ontario, Canada. \\ *Correspondence to: lbarriau@uottawa.ca
}

\section{Table of Contents}

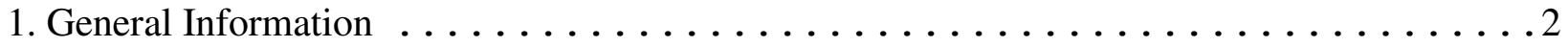

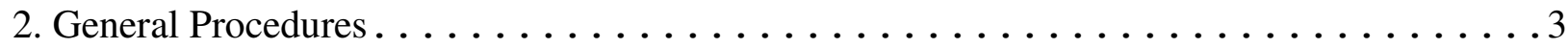

GP1- Preparation of Biarenes from Iodoarenes using $\left[\mathrm{Au}_{2}(\mathrm{dppm})_{2}\right] \mathrm{Cl}_{2} \ldots \ldots \ldots$

GP2- Preparation of Homocoupled Bromoalkanes with $\left[\mathrm{Au}_{2}(\mathrm{dppm})_{2}\right] \mathrm{Cl}_{2} \ldots \ldots . \ldots 3$

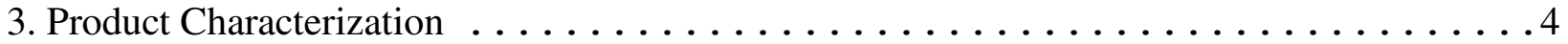

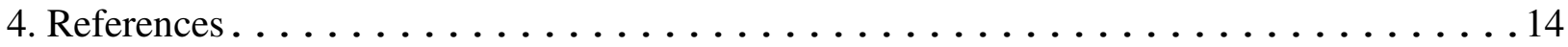

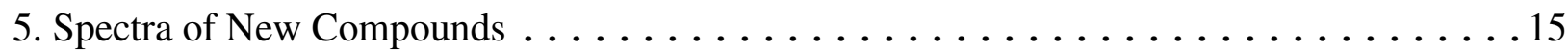

6. ${ }^{1} \mathrm{H}$ NMR Spectra of Known Compounds $\ldots \ldots \ldots \ldots \ldots \ldots \ldots \ldots \ldots \ldots \ldots \ldots$ 


\section{General Information}

All reactions were performed under argon atmosphere Pyrex glassware equipped with a magnetic stir bar, capped with a septum, unless otherwise indicated. All commercial reagents were used without further purification, unless otherwise noted. Reactions were monitored by thin layer chromatography (TLC) analysis. TLC plates were viewed under UV light and stained with potassium permanganate or p-anisaldehyde staining solution. Yields refer to products isolated after purification, unless otherwise stated. Proton nuclear magnetic resonance (1H NMR) spectra were recorded on a Bruker AMX $400 \mathrm{MHz}$. NMR samples were dissolved in chloroform-d (unless specified otherwise) and chemical shifts are reported in ppm referenced to residual undeuterated solvent. Data are reported as follows: chemical shift, multiplicity, coupling, integration, where multiplicity is as follows : $\mathrm{s}=$ singlet, $\mathrm{d}=$ doublet, $\mathrm{dd}=$ doublet of doublets, ddd $=$ doublet of doublets of doublets, $\mathrm{dt}=$ doublet of triplets, $\mathrm{ddt}=$ doublet of doublets of triplets, $\mathrm{dq}=$ doublet of quartets, dquin $=$ doublet of quintets, $\mathrm{br}=$ broad signal, $\mathrm{t}=$ triplet, $\mathrm{td}=$ triplet of doublets, $\mathrm{tt}=$ triplet of triplets, $\mathrm{tq}=$ triplet of quartets, tquin $=$ triplet of quintets, $\mathrm{q}=$ quartet, qd $=$ quartet of doublets, quin $=$ quintet, $\mathrm{spt}=$ septet, $\mathrm{m}=$ multiplet, or otherwise noted. Carbon nuclear magnetic resonance (13C NMR) spectra were recorded on the same Bruker instruments as in proton NMR using $75 \mathrm{MHz}$ or $101 \mathrm{MHz}$. IR spectra were recorded with an Agilent Technologies Cary 630 FTIR Spectrometer equipped with a diamond ATR module. HRMS were obtained on a Kratos Analytical Concept instrument (University of Ottawa Mass Spectrum Centre). 


\section{General Procedures}

General Procedure 1 (GP1). Preparation of Biarenes from Iodoarenes using $\left[\mathrm{Au}_{2}(\mathrm{dppm})_{2}\right] \mathrm{Cl}_{2}$. To an oven dried $8 \mathrm{~mL}$ Pyrex screw-top reaction vessel was added the iodoarene $(0.2 \mathrm{mmol}, 1.0$ equiv.), $\left[\mathrm{Au}_{2}(\mathrm{dppm})_{2}\right] \mathrm{Cl}_{2}(0.01 \mathrm{mmol}, 0.05$ equiv. $), \mathrm{K}_{2} \mathrm{HPO}_{4}(0.2 \mathrm{mmol}, 1.0$ equiv.), degassed $\mathrm{MeCN}$ and degassed $\mathrm{MeOH}$ (1:1, $400 \mu \mathrm{L}$ total, $0.5 \mathrm{M})$. Triethylamine $(0.2 \mathrm{mmol}, 1.0$ equiv.) was then added to the solution. The reaction vessel was capped and back-filled with argon for 30 seconds, then irradiated with a UVA (365 nm) LED at an approximate distance of $5 \mathrm{~mm}$ for 16 hours. The resulting mixture was concentrated and the crude residue was further purified by flash chromatography (0-100\% EtOAc:Hexanes), where relevant fractions were combined, concentrated and characterized by proton and carbon NMR (400 and $101 \mathrm{MHz}$, respectively), HR-MS, and IR.

General Procedure 2 (GP2). Preparation of Homocoupled Bromoalkanes with $\left[\mathrm{Au}_{2}(\mathrm{dppm})_{2}\right] \mathrm{Cl}_{2}$. To an oven dried $8 \mathrm{~mL}$ Pyrex screw-top reaction vessel was added the bromoalkane ( $0.4 \mathrm{mmol}, 1.0$ equiv.), $\left[\mathrm{Au}_{2}(\mathrm{dppm})_{2}\right] \mathrm{Cl}_{2}\left(0.02 \mathrm{mmol}, 0.05\right.$ equiv.), $\mathrm{CD}_{3} \mathrm{CN}$ and $\mathrm{CD}_{3} \mathrm{OD}(1: 1,800 \mu \mathrm{L}$ total, $0.5 \mathrm{M})$, and $\mathrm{d}_{15}$-Triethylamine $(0.4 \mathrm{mmol}, 1.0$ equiv., added after sparging). The reaction vessel was capped, degassed with argon by sparging for 5 minutes, then irradiated with a UVA $(365 \mathrm{~nm})$ LED at an approximate distance of $5 \mathrm{~mm}$ for 16 hours. The resulting mixture was concentrated and the crude residue was further purified by flash chromatography (0-100\% EtOAc:Hexanes), where relevant fractions were combined, concentrated and characterized by proton and carbon NMR (400 and $101 \mathrm{MHz}$, respectively), HR-MS, and IR. 


\section{Product Characterization}

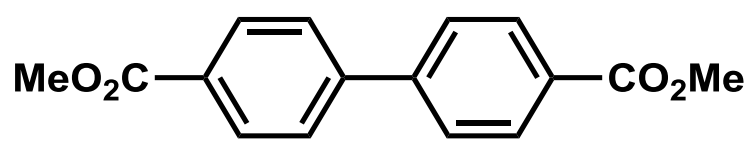

dimethyl [1,1'-biphenyl]-4,4'-dicarboxylate (2a)

Synthesized according to GP1, affording $25 \mathrm{mg}(91 \%)$ of an amorphous solid, characterized according to NMR comparison. ${ }^{1}$

${ }^{1}$ H NMR $\left(400 \mathrm{MHz}, \mathrm{CDCl}_{3}\right) \delta=8.14(\mathrm{dt}, J=8.4,1.9 \mathrm{~Hz}, 4 \mathrm{H}), 7.70(\mathrm{dt}, J=8.4,1.8 \mathrm{~Hz}, 4 \mathrm{H})$, 3.96 (s, $6 \mathrm{H}$ ) ppm. ${ }^{13} \mathrm{C}$ NMR (101 MHz, $\left.\mathrm{CDCl}_{3}\right) \delta=166.8$ (2 X C), 144.3 (2 X C), 130.2 (4 X $\mathrm{CH}), 129.7$ (2 X C), $127.2(4 \mathrm{X} \mathrm{CH}), 52.2\left(2 \mathrm{X} \mathrm{CH}_{3}\right) \mathrm{ppm}$.

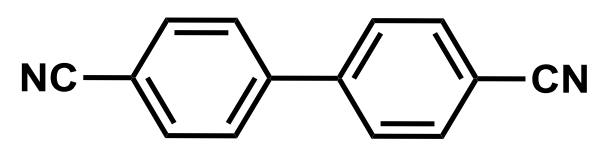

\section{[1,1'-biphenyl]-4,4'-dicarbonitrile (2b)}

Synthesized according to GP1, affording $12 \mathrm{mg}(60 \%)$ of an amorphous solid, characterized according to NMR comparison. ${ }^{2}$

${ }^{1} \mathbf{H}$ NMR $\left(400 \mathrm{MHz}, \mathrm{CDCl}_{3}\right) \delta=7.79-7.73(\mathrm{~m}, 4 \mathrm{H}), 7.71-7.64(\mathrm{~m}, 4 \mathrm{H}) \mathrm{ppm} .{ }^{13} \mathbf{C}$ NMR $(101$ $\left.\mathrm{MHz}, \mathrm{CDCl}_{3}\right) \delta=143.5$ ( 2 X C), $132.9(4 \mathrm{X} \mathrm{CH}), 128.0(4 \mathrm{X} \mathrm{CH}), 118.4$ (2 X C), 112.5 (2 X C) ppm.

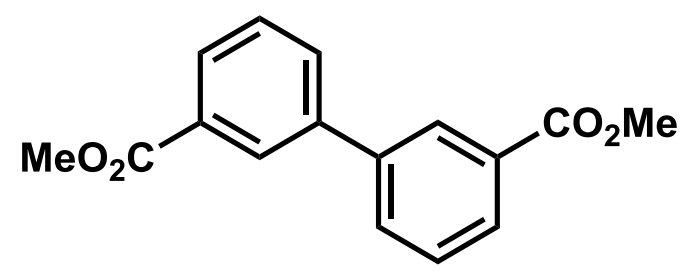

dimethyl [1,1'-biphenyl]-3,3'-dicarboxylate (2c)

Synthesized according to GP1, affording $18 \mathrm{mg}(66 \%)$ of an amorphous solid, characterized according to NMR comparison. ${ }^{1}$

${ }^{1} \mathbf{H}$ NMR $\left(400 \mathrm{MHz}, \mathrm{CDCl}_{3}\right) \delta=8.31(\mathrm{t}, J=1.7 \mathrm{~Hz}, 2 \mathrm{H}), 8.06(\mathrm{dt}, J=7.8,1.3 \mathrm{~Hz}, 2 \mathrm{H}), 7.85-$ $7.80(\mathrm{~m}, 2 \mathrm{H}), 7.55(\mathrm{t}, J=7.7 \mathrm{~Hz}, 2 \mathrm{H}), 3.97(\mathrm{~s}, 6 \mathrm{H}) \mathrm{ppm} .{ }^{13} \mathbf{C} \mathbf{N M R}\left(101 \mathrm{MHz}, \mathrm{CDCl}_{3}\right) \delta=$ 166.9 ( 2 X C), 140.4 ( 2 X C), 131.5 ( 2 X CH), 130.9 ( 2 X C), 129.0 ( 2 X CH), 128.8 ( 2 X CH), $128.3(2 \mathrm{X} \mathrm{CH}), 52.3\left(2 \mathrm{X} \mathrm{CH}_{3}\right) \mathrm{ppm}$. 


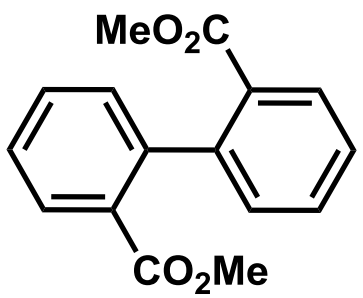

dimethyl [1,1'-biphenyl]-2,2'-dicarboxylate (2d)

Synthesized according to GP1, affording $4 \mathrm{mg}(14 \%)$ of an amorphous solid, characterized according to NMR comparison. ${ }^{1}$

${ }^{1} \mathbf{H}$ NMR $\left(400 \mathrm{MHz}, \mathrm{CDCl}_{3}\right) \delta=8.02(\mathrm{dd}, J=7.8,1.4 \mathrm{~Hz}, 2 \mathrm{H}), 7.55(\mathrm{td}, J=7.5,1.5 \mathrm{~Hz}, 2 \mathrm{H})$, $7.44(\mathrm{td}, J=7.6,1.3 \mathrm{~Hz}, 2 \mathrm{H}), 7.22(\mathrm{dd}, J=7.5,1.2 \mathrm{~Hz}, 2 \mathrm{H}), 3.63(\mathrm{~s}, 6 \mathrm{H}) \mathrm{ppm} .{ }^{13} \mathbf{C}$ NMR $(101$ $\left.\mathrm{MHz}, \mathrm{CDCl}_{3}\right) \delta=167.4(2 \mathrm{X} \mathrm{C}), 143.3(2 \mathrm{X} \mathrm{C}), 131.4(2 \mathrm{X} \mathrm{CH}), 130.2(2 \mathrm{X} \mathrm{CH}), 129.8(2 \mathrm{X}$ $\mathrm{CH}), 129.4(2 \mathrm{X} \mathrm{C}), 127.2(2 \mathrm{X} \mathrm{CH}), 51.8\left(2 \mathrm{X} \mathrm{CH}_{3}\right) \mathrm{ppm}$.

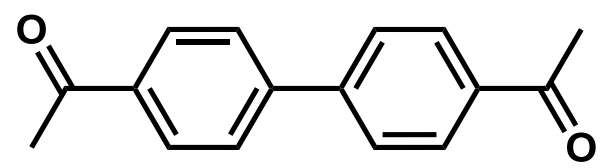

1,1'-([1,1'-biphenyl]-4,4'-diyl)bis(ethan-1-one) (2e)

Synthesized according to GP1, affording $15 \mathrm{mg}(63 \%)$ of an amorphous solid, characterized according to NMR comparison. ${ }^{1}$

${ }^{1} \mathbf{H}$ NMR $\left(400 \mathrm{MHz}, \mathrm{CDCl}_{3}\right) \delta=8.08-8.00(\mathrm{~m}, 4 \mathrm{H}), 7.74-7.67(\mathrm{~m}, 4 \mathrm{H}), 2.63(\mathrm{~s}, 6 \mathrm{H}) \mathrm{ppm} .{ }^{13} \mathbf{C}$ NMR $\left(101 \mathrm{MHz}, \mathrm{CDCl}_{3}\right) \delta=197.6$ ( 2 X C), 144.3 ( 2 X C), 136.6 (2 X C), $129.0(4 \mathrm{X} \mathrm{CH})$, $127.5(4 \mathrm{X} \mathrm{CH}), 26.7\left(2 \mathrm{X} \mathrm{CH}_{3}\right) \mathrm{ppm}$.

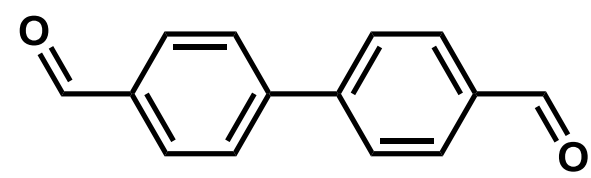

\section{[1,1'-biphenyl]-4,4'-dicarbaldehyde (2f)}

Synthesized according to GP1, affording $12 \mathrm{mg}$ (59\%) of an amorphous solid, characterized according to NMR comparison. ${ }^{1}$

${ }^{1} \mathbf{H}$ NMR $\left(400 \mathrm{MHz}, \mathrm{CDCl}_{3}\right) \delta=10.10(\mathrm{~s}, 2 \mathrm{H}), 8.04-7.97(\mathrm{~m}, 4 \mathrm{H}), 7.85-7.78(\mathrm{~m}, 4 \mathrm{H}) \mathrm{ppm} .{ }^{13} \mathbf{C}$ NMR $\left(101 \mathrm{MHz}, \mathrm{CDCl}_{3}\right) \delta=191.7(2 \mathrm{X} \mathrm{CH}), 145.6(2 \mathrm{X} \mathrm{C}), 136.0(2 \mathrm{X} \mathrm{C}), 130.4(4 \mathrm{X} \mathrm{CH})$, $128.0(4 \mathrm{X} \mathrm{CH}) \mathrm{ppm}$.

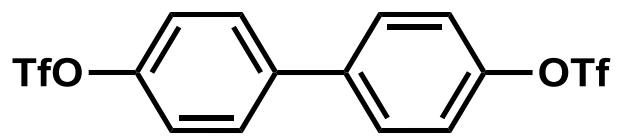

\section{[1,1'-biphenyl]-4,4'-diyl bis(trifluoromethanesulfonate) (2g)}

Synthesized according to GP1, affording $26 \mathrm{mg}(57 \%)$ of an amorphous solid.

IR (neat, $\mathbf{~ c m}^{-1}$ ): 1421(s), 1204(vs), 1134(vs), 878(s), 829(s), 743(s). ${ }^{1} \mathbf{H}$ NMR (400 MHz, $\left.\mathrm{CDCl}_{3}\right) \delta=7.63(\mathrm{~s}, 4 \mathrm{H}), 7.41-7.37(\mathrm{~m}, 4 \mathrm{H}) \mathrm{ppm} .{ }^{13} \mathbf{C ~ N M R}\left(101 \mathrm{MHz}, \mathrm{CDCl}_{3}\right) \delta=149.4(2 \mathrm{X}$ C), 139.7 (2 X C), $129.0(4 \mathrm{X} \mathrm{CH}), 122.0(4 \mathrm{X} \mathrm{CH}), 118.8$ (q, $J=321.3 \mathrm{~Hz}, 2$ X C) ppm. HRMS (EI) $\mathrm{m} / \mathrm{z}$ calc'd for $\mathrm{C}_{14} \mathrm{H}_{8} \mathrm{~F}_{6} \mathrm{O}_{6} \mathrm{~S}_{2}\left[\mathrm{M}^{+}\right] 449.9666$ found 449.9700 . 


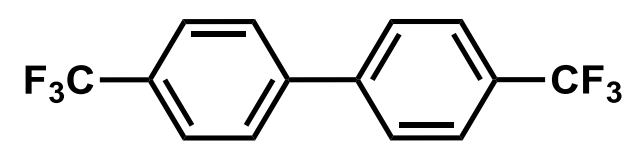

4,4'-bis(trifluoromethyl)-1,1'-biphenyl (2h)

Synthesized according to GP1, affording $18 \mathrm{mg}(61 \%)$ of an amorphous solid, characterized according to NMR comparison. ${ }^{1}$

${ }^{\mathbf{1}} \mathbf{H}$ NMR $\left(400 \mathrm{MHz}, \mathrm{CDCl}_{3}\right) \delta=7.78-7.69(\mathrm{~m}, 8 \mathrm{H}) \mathrm{ppm} .{ }^{\mathbf{1 3}} \mathbf{C} \mathbf{N M R}\left(101 \mathrm{MHz}, \mathrm{CDCl}_{3}\right) \delta=$ 143.2 (2 X C), 130.3 (q, $J=32.6 \mathrm{~Hz}, 2$ X C), 127.6 (4 X CH), 125.9 (q, $J=3.8 \mathrm{~Hz}, 4 \mathrm{X} \mathrm{CH}$ ), $124.1(\mathrm{q}, J=272.2 \mathrm{~Hz}, 2 \mathrm{X} \mathrm{C}) \mathrm{ppm}$.

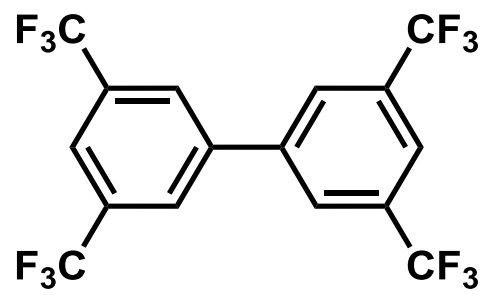

3,3',5,5'-tetrakis(trifluoromethyl)-1,1'-biphenyl (2i)

Synthesized according to GP1, affording $15 \mathrm{mg}$ (35\%) of an amorphous solid, characterized according to NMR comparison. ${ }^{3}$

${ }^{1} \mathbf{H}$ NMR $\left(400 \mathrm{MHz}, \mathrm{CDCl}_{3}\right) \delta=8.01(\mathrm{~s}, 4 \mathrm{H}), 7.97$ (s, $\left.2 \mathrm{H}\right) \mathrm{ppm} .{ }^{\mathbf{1 3}} \mathbf{C ~ N M R}\left(101 \mathrm{MHz}, \mathrm{CDCl}_{3}\right)$ $\delta=140.4(2 \mathrm{X} \mathrm{C}), 132.9$ (q, $J=34.5 \mathrm{~Hz}, 4 \mathrm{X} \mathrm{C}), 127.5$ (d, $J=2.9 \mathrm{~Hz}, 4 \mathrm{X} \mathrm{CH}), 122.6$ (spt, $J=$ $3.7 \mathrm{~Hz}, 2 \mathrm{X} \mathrm{CH}), 123.0(\mathrm{q}, J=272.2 \mathrm{~Hz}, 4 \mathrm{X} \mathrm{C}) \mathrm{ppm}$.

\section{4,4'-dichloro-1,1'-biphenyl (2j)}

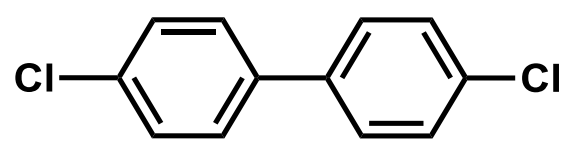

Synthesized according to GP1, affording $12 \mathrm{mg}$ (52\%) of an amorphous solid, characterized according to NMR comparison. ${ }^{4}$

${ }^{1} \mathbf{H}$ NMR $\left(400 \mathrm{MHz}, \mathrm{CDCl}_{3}\right) \delta=7.52-7.46$ (m, $\left.4 \mathrm{H}\right), 7.45-7.39$ (m, $\left.4 \mathrm{H}\right)$ ppm. ${ }^{13} \mathbf{C}$ NMR $(101$ $\left.\mathrm{MHz}, \mathrm{CDCl}_{3}\right) \delta=138.4$ (2 X C), 133.8 (2 X C), 129.1 (4 X CH), 128.2 (4 X CH) ppm.

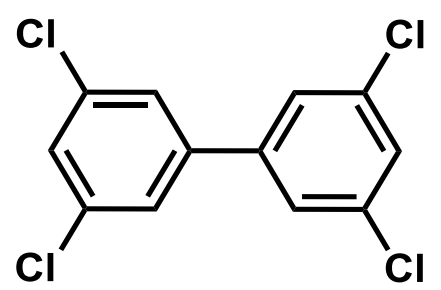

\section{3,3',5,5'-tetrachloro-1,1'-biphenyl (2k)}

Synthesized according to GP1, affording $15 \mathrm{mg}(52 \%)$ of an amorphous solid, characterized according to NMR comparison. ${ }^{5}$

${ }^{1} \mathbf{H}$ NMR $\left(400 \mathrm{MHz}, \mathrm{CDCl}_{3}\right) \delta=7.44-7.38(\mathrm{~m}, 6 \mathrm{H}) \mathrm{ppm} .{ }^{13} \mathbf{C}$ NMR $\left(101 \mathrm{MHz}, \mathrm{CDCl}_{3}\right) \delta=$ 141.4 (2 X C), 135.7 (4 X C), 128.4 (2 X CH), 125.6 (4 X CH) ppm. 


\section{4,4'-dibromo-1,1'-biphenyl (2I)}

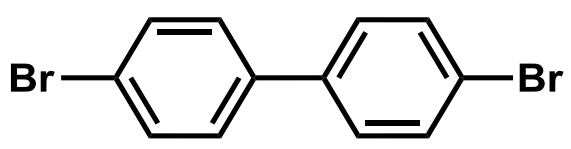

Synthesized according to GP1, affording $10 \mathrm{mg}(31 \%)$ of an amorphous solid, characterized according to NMR comparison. ${ }^{1}$

${ }^{1} \mathbf{H}$ NMR $\left(400 \mathrm{MHz}, \mathrm{CDCl}_{3}\right) \delta=7.60-7.54(\mathrm{~m}, 4 \mathrm{H}), 7.46-7.39$ (m, $\left.4 \mathrm{H}\right) \mathrm{ppm} .{ }^{13} \mathbf{C}$ NMR $(101$ $\left.\mathrm{MHz}, \mathrm{CDCl}_{3}\right) \delta=138.9$ (2 X C), $132.0(4 \mathrm{X} \mathrm{CH}), 128.5$ (4 X CH), 122.0 (2 X C) ppm.

\section{3,3'-dinitro-1,1'-biphenyl (2m)}

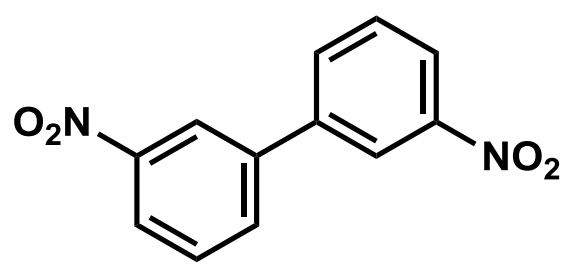

Synthesized according to GP1, affording $10 \mathrm{mg}$ (39\%) of an amorphous solid, characterized according to NMR comparison. ${ }^{6}$

${ }^{1} \mathbf{H}$ NMR $\left(400 \mathrm{MHz}, \mathrm{CDCl}_{3}\right) \delta=8.48(\mathrm{t}, J=2.0 \mathrm{~Hz}, 2 \mathrm{H}), 8.31-8.25(\mathrm{~m}, 2 \mathrm{H}), 7.99-7.92(\mathrm{~m}, 2$ $\mathrm{H}), 7.69$ (t, $J=8.0 \mathrm{~Hz}, 2 \mathrm{H}) \mathrm{ppm} .{ }^{13} \mathbf{C ~ N M R}\left(101 \mathrm{MHz}, \mathrm{CDCl}_{3}\right) \delta=148.9(2 \mathrm{X} \mathrm{C}), 140.3(2 \mathrm{X}$ C), $133.0(2 \mathrm{X} \mathrm{CH}), 130.3(2 \mathrm{X} \mathrm{CH}), 123.3(2 \mathrm{X} \mathrm{CH}), 122.1(2 \mathrm{X} \mathrm{CH}) \mathrm{ppm}$.

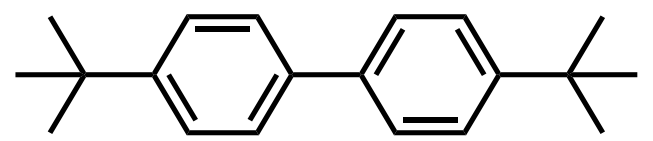

\section{4,4'-di-tert-butyl-1,1'-biphenyl (2o)}

Synthesized according to GP1, affording $14 \mathrm{mg}$ (53\%) of an amorphous solid, characterized according to NMR comparison. ${ }^{7}$

${ }^{1} \mathbf{H}$ NMR $\left(400 \mathrm{MHz}, \mathrm{CDCl}_{3}\right) \delta=7.55(\mathrm{dt}, J=8.4,2.0 \mathrm{~Hz}, 4 \mathrm{H}), 7.47(\mathrm{dt}, J=8.3,1.7 \mathrm{~Hz}, 4 \mathrm{H})$, $1.38(\mathrm{~s}, 18 \mathrm{H}) \mathrm{ppm} .{ }^{13} \mathrm{C} \mathrm{NMR}\left(101 \mathrm{MHz}, \mathrm{CDCl}_{3}\right) \delta=149.9$ (2 X C), 138.2 (2 X C), 126.6 (4 X $\mathrm{CH}), 125.6$ (4 X CH), 34.5 (2 X C), $31.4\left(6 \mathrm{X} \mathrm{CH}_{3}\right) \mathrm{ppm}$.

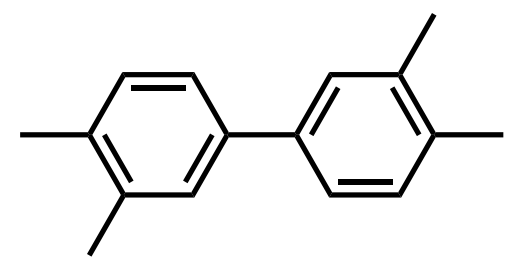

\section{$3,3^{\prime}, 4,4^{\prime}$ '-tetramethyl-1,1'-biphenyl (2p)}

Synthesized according to GP1, affording $10 \mathrm{mg}$ (47\%) of an amorphous solid, characterized according to NMR comparison. ${ }^{6}$

${ }^{1}$ H NMR $\left(400 \mathrm{MHz}, \mathrm{CDCl}_{3}\right) \delta=7.37-7.33(\mathrm{~m}, 2 \mathrm{H}), 7.31(\mathrm{dd}, J=7.6,1.8 \mathrm{~Hz}, 2 \mathrm{H}), 7.17(\mathrm{~d}, J=$ $7.8 \mathrm{~Hz}, 2 \mathrm{H}), 2.32$ (s, $6 \mathrm{H}), 2.29$ (s, $6 \mathrm{H}) \mathrm{ppm} .{ }^{13} \mathbf{C ~ N M R}\left(101 \mathrm{MHz}, \mathrm{CDCl}_{3}\right) \delta=138.9(2 \mathrm{X} \mathrm{C})$, 136.8 ( 2 X C), 135.3 (2 X C), 130.0 (2 X CH), 128.3 ( 2 X CH), $124.4(2 \mathrm{X} \mathrm{CH}), 19.9\left(2 \mathrm{X} \mathrm{CH}_{3}\right)$, $19.4\left(2 \mathrm{X} \mathrm{CH}_{3}\right) \mathrm{ppm}$. 


\section{3,3 ',5,5'-tetramethyl-1,1'-biphenyl (2q)}

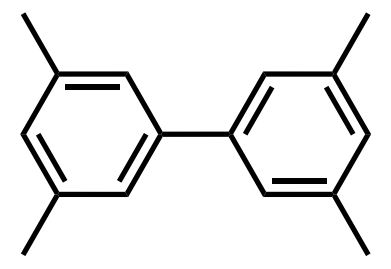

Synthesized according to GP1, affording $7 \mathrm{mg}(34 \%)$ of an amorphous solid, characterized according to NMR comparison. ${ }^{8}$

${ }^{1} \mathbf{H ~ N M R}\left(400 \mathrm{MHz}, \mathrm{CDCl}_{3}\right) \delta=7.18(\mathrm{~s}, 4 \mathrm{H}), 6.96(\mathrm{~s}, 2 \mathrm{H}), 2.36(\mathrm{~s}, 6 \mathrm{H}), 2.36(\mathrm{~s}, 6 \mathrm{H}) \mathrm{ppm} .{ }^{13} \mathbf{C}$ NMR $\left(101 \mathrm{MHz}, \mathrm{CDCl}_{3}\right) \delta=141.5$ ( 2 X C), 138.1 (4 X C), $128.7(2 \mathrm{X} \mathrm{CH}), 125.1(4 \mathrm{X} \mathrm{CH})$, $21.4\left(4 \mathrm{X} \mathrm{CH}_{3}\right) \mathrm{ppm}$.

\section{4,4'-diethoxy-1,1'-biphenyl (2r)}

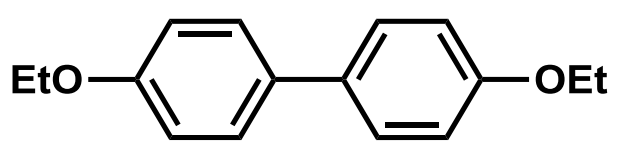

Synthesized according to GP1, affording $7 \mathrm{mg}$ (29\%) of an amorphous solid, characterized according to NMR comparison. ${ }^{9}$

${ }^{1}$ H NMR $\left(400 \mathrm{MHz}, \mathrm{CDCl}_{3}\right) \delta=7.50-7.45(\mathrm{~m}, 4 \mathrm{H}), 6.99-6.92(\mathrm{~m}, 4 \mathrm{H}), 4.08(\mathrm{q}, J=7.0 \mathrm{~Hz}, 4$ $\mathrm{H}), 1.45(\mathrm{t}, J=7.0 \mathrm{~Hz}, 6 \mathrm{H}) \mathrm{ppm} .{ }^{13} \mathbf{C}$ NMR $\left(101 \mathrm{MHz}, \mathrm{CDCl}_{3}\right) \delta=158.0(2 \mathrm{X} \mathrm{C}), 133.4(2 \mathrm{X}$ C), $127.7(4 \mathrm{X} \mathrm{CH}), 114.7(4 \mathrm{X} \mathrm{CH}), 63.5\left(2 \mathrm{X} \mathrm{CH}_{2}\right), 14.9\left(2 \mathrm{X} \mathrm{CH}_{3}\right) \mathrm{ppm}$.<smiles>N[C@@H](Cc1ccc(I)cc1)C(=O)O</smiles>
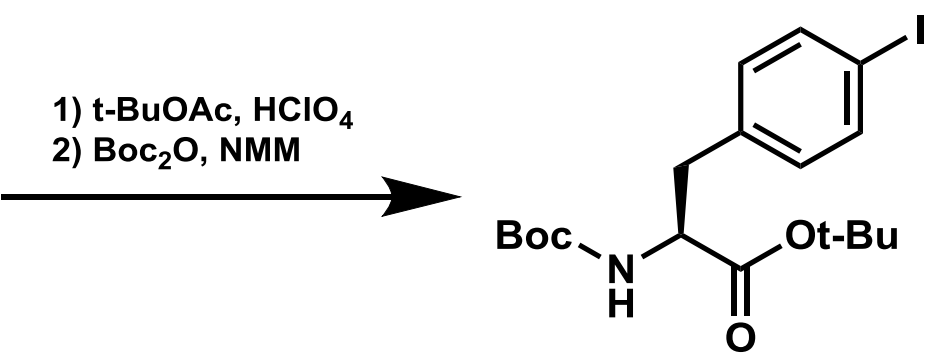

tert-butyl (S)-2-((tert-butoxycarbonyl)amino)-3-(4-iodophenyl)propanoate (1s)

Synthesized according to literature procedure and characterized according to NMR comparison. ${ }^{10}$

${ }^{1} \mathbf{H}$ NMR $\left(400 \mathrm{MHz}, \mathrm{CDCl}_{3}\right) \delta=7.60-7.58(\mathrm{~m}, 2 \mathrm{H}), 6.91-6.89(\mathrm{~m}, 2 \mathrm{H}), 4.96(\mathrm{~d}, J=7.5 \mathrm{~Hz}, 1 \mathrm{H})$, $4.40(\mathrm{q}, J=6.8 \mathrm{~Hz}, 1 \mathrm{H}), 3.03-2.92(\mathrm{~m}, 2 \mathrm{H}), 1.40(\mathrm{~s}, 9 \mathrm{H}), 1.39(\mathrm{~s}, 9 \mathrm{H}) \mathrm{ppm} .{ }^{13} \mathbf{C}$ NMR $(101$ $\left.\mathrm{MHz}, \mathrm{CDCl}_{3}\right) \delta=170.6(\mathrm{C}), 155.0(\mathrm{C}), 137.4(2 \mathrm{X} \mathrm{CH}), 136.1(\mathrm{C}), 131.6(2 \mathrm{X} \mathrm{CH}), 92.2(\mathrm{C})$, $82.3(\mathrm{C}), 79.8(\mathrm{C}), 54.6(\mathrm{CH}), 38.0\left(\mathrm{CH}_{2}\right), 28.3\left(3 \mathrm{X} \mathrm{CH}_{3}\right), 28.0\left(3 \mathrm{X} \mathrm{CH}_{3}\right) \mathrm{ppm}$. 


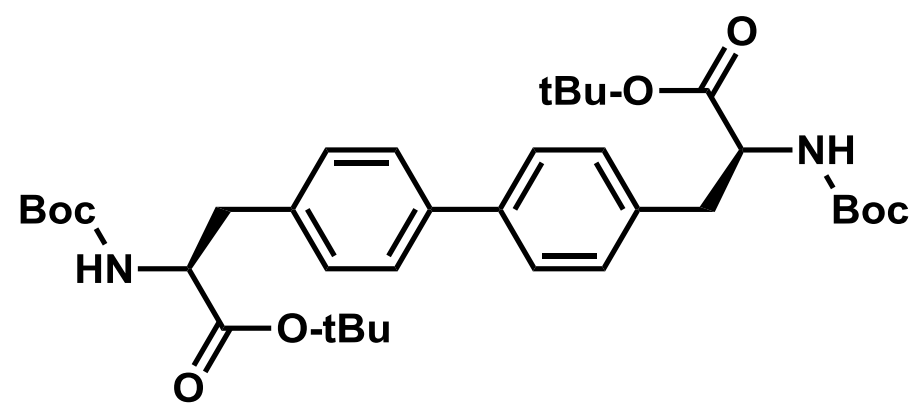

di-tert-butyl 3,3'-([1,1'-biphenyl]-4,4'-diyl)(2S,2'S)-bis(2-((tertbutoxycarbonyl)amino)propanoate) (2s)

Synthesized according to GP1 on a $0.181 \mathrm{mmol}$ scale, affording $38 \mathrm{mg}(66 \%)$ of an amorphous solid.

IR (neat, $\left.\mathbf{c m}^{\mathbf{- 1}}\right)$ : 3441(br), 2977(m), 2932(m), 1712(vs), 1709(vs), 1702(vs), 1698(vs), 1497(m), 1366(s), 701 (vs). ${ }^{1} \mathbf{H}$ NMR $\left(400 \mathrm{MHz}, \mathrm{CDCl}_{3}\right) \delta=7.47$ (d, $\left.J=8.0 \mathrm{~Hz}, 2 \mathrm{H}\right), 7.28-7.14(\mathrm{~m}, 6 \mathrm{H})$, 5.02-4.73 (m, $2 \mathrm{H}), 4.48-4.27(\mathrm{~m}, 2 \mathrm{H}), 3.05(\mathrm{dd}, J=16.1,5.9 \mathrm{~Hz}, 4 \mathrm{H}), 1.40(\mathrm{~s}, 9 \mathrm{H}), 1.40$ (s, 9 $\mathrm{H}), 1.39$ (s, $9 \mathrm{H}), 1.37$ (s, $9 \mathrm{H}) \mathrm{ppm} .{ }^{13} \mathrm{C} \mathbf{N M R}\left(101 \mathrm{MHz}, \mathrm{CDCl}_{3}\right) \delta=171.9$ (2 X C), $155.1(2 \mathrm{X}$ C), 139.3 (2 X C), $136.4(\mathrm{C}), 135.4(\mathrm{C}), 129.9$ (2 X CH), 129.5 (2 X CH), 128.3 (2 X CH), 126.8 $(\mathrm{CH}), 126.8(\mathrm{CH}), 82.1(\mathrm{C}), 82.0(\mathrm{C}), 79.6(\mathrm{C}), 79.6(\mathrm{C}), 54.8(2 \mathrm{X} \mathrm{CH}), 38.5\left(\mathrm{CH}_{2}\right), 38.2\left(\mathrm{CH}_{2}\right)$, $28.3\left(6 \mathrm{X} \mathrm{CH}_{3}\right), 27.9\left(3 \mathrm{X} \mathrm{CH}_{3}\right), 27.9\left(3 \mathrm{X} \mathrm{CH}_{3}\right) \mathrm{ppm}$. HRMS (ESI) m/z calc'd for $\mathrm{C}_{36} \mathrm{H}_{52} \mathrm{~N}_{2} \mathrm{O}_{8}$ $\left[\mathrm{M}^{+}\right]$640.3724, found $[\mathrm{M}+23] 663.3621$.

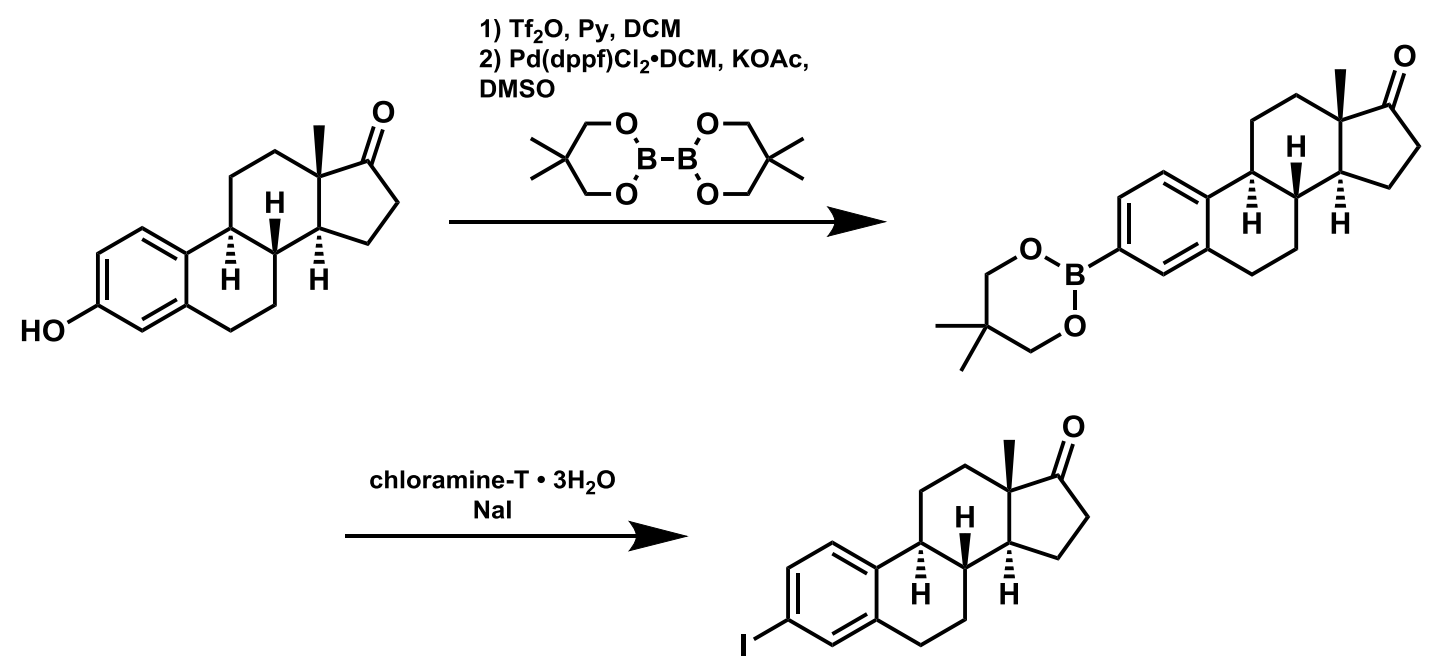

(8R,9S,13S,14S)-3-iodo-13-methyl-6,7,8,9,11,12,13,14,15,16-decahydro-17Hcyclopenta[a]phenanthren-17-one (1t)

Synthesized according to literature procedure and characterized according to NMR comparison. $^{11}$

${ }^{1}$ H NMR $\left(400 \mathrm{MHz}, \mathrm{CDCl}_{3}\right) \delta=7.50-7.43(\mathrm{~m}, 2 \mathrm{H}), 7.03(\mathrm{~d}, J=8.0 \mathrm{~Hz}, 1 \mathrm{H}), 2.88(\mathrm{dd}, J=8.7$, $4.1 \mathrm{~Hz}, 2 \mathrm{H}), 2.52$ (dd, $J=18.8,8.5 \mathrm{~Hz}, 1 \mathrm{H}), 2.42-2.34(\mathrm{~m}, 1 \mathrm{H}), 2.30-2.21(\mathrm{~m}, 1 \mathrm{H}), 2.21-1.94$ $(\mathrm{m}, 4 \mathrm{H}), 1.70-1.36(\mathrm{~m}, 6 \mathrm{H}), 0.91(\mathrm{~s}, 3 \mathrm{H}) \mathrm{ppm} .{ }^{13} \mathrm{C} \mathrm{NMR}\left(101 \mathrm{MHz}, \mathrm{CDCl}_{3}\right) \delta=220.5(\mathrm{C})$, $139.4(\mathrm{C}), 139.1(\mathrm{C}), 137.6(\mathrm{CH}), 134.6(\mathrm{CH}), 127.3(\mathrm{CH}), 91.1(\mathrm{CH}), 50.3(\mathrm{CH}), 47.8(\mathrm{C}), 44.1$ $(\mathrm{CH}), 37.7(\mathrm{CH}), 35.7\left(\mathrm{CH}_{2}\right), 31.4\left(\mathrm{CH}_{2}\right), 28.9\left(\mathrm{CH}_{2}\right), 26.1\left(\mathrm{CH}_{2}\right), 25.4\left(\mathrm{CH}_{2}\right), 21.5\left(\mathrm{CH}_{2}\right), 13.7$ $\left(\mathrm{CH}_{3}\right) \mathrm{ppm}$. 


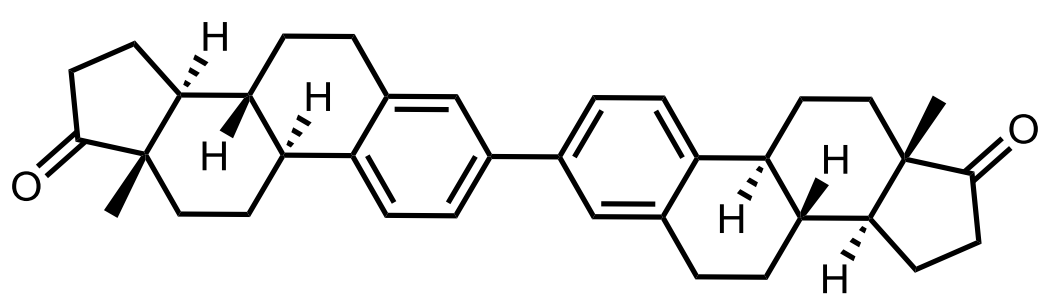

estrone dimer (2t)

Synthesized according to GP1, affording $14 \mathrm{mg}$ (28\%) of an amorphous solid.

IR (neat, $\mathbf{~ c m}^{-1}$ ): 2928(m), 2863(m), 1737(vs). ${ }^{1} \mathbf{H}$ NMR (400 MHz, $\left.\mathrm{CDCl}_{3}\right) \delta=7.41-7.35(\mathrm{~m}, 4$ H), $7.33(\mathrm{~s}, 2 \mathrm{H}), 3.00(\mathrm{dd}, J=8.8,4.0 \mathrm{~Hz}, 4 \mathrm{H}), 2.56-2.46(\mathrm{~m}, 4 \mathrm{H}), 2.37(\mathrm{td}, J=10.7,3.6 \mathrm{~Hz}, 2$ $\mathrm{H}), 2.21-1.98(\mathrm{~m}, 8 \mathrm{H}), 1.71-1.47(\mathrm{~m}, 12 \mathrm{H}), 0.94(\mathrm{~s}, 6 \mathrm{H}) \mathrm{ppm} .{ }^{13} \mathbf{C}$ NMR $\left(101 \mathrm{MHz}, \mathrm{CDCl}_{3}\right) \delta$ = 220.89 (2 X C), 138.7 (2 X C), 138.6 (2 X C), 136.8 (2 X C), 127.5 (2 X CH), 125.7 (2 X CH), $124.4(2 \mathrm{X} \mathrm{CH}), 50.5(2 \mathrm{X} \mathrm{CH}), 48.0(2 \mathrm{X} \mathrm{C}), 44.4(2 \mathrm{X} \mathrm{CH}), 38.2(2 \mathrm{X} \mathrm{CH}), 35.8\left(2 \mathrm{X} \mathrm{CH}_{2}\right)$, $31.6\left(2 \mathrm{X} \mathrm{CH}_{2}\right), 29.5\left(2 \mathrm{X} \mathrm{CH}_{2}\right), 26.5\left(2 \mathrm{X} \mathrm{CH}_{2}\right), 25.7\left(2 \mathrm{X} \mathrm{CH}_{2}\right), 21.6\left(2 \mathrm{X} \mathrm{CH}_{2}\right), 13.8(2 \mathrm{X}$ $\left.\mathrm{CH}_{3}\right) \mathrm{ppm}$. HRMS (EI) m/z calc'd for $\mathrm{C}_{36} \mathrm{H}_{42} \mathrm{O}_{2}\left[\mathrm{M}^{+}\right]$506.3185, found 506.3205.

\section{N-phenylacetamide (3n)}

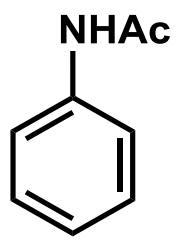

Synthesized according to GP1, affording $23 \mathrm{mg}(85 \%)$ of an amorphous solid, characterized according to NMR comparison. ${ }^{12}$

${ }^{1}$ H NMR (400 MHz, $\left.\left(\mathrm{CD}_{3}\right)_{2} \mathrm{CO}\right): \delta=9.13$ (br. s., $\left.1 \mathrm{H}\right), 7.63(\mathrm{~d}, J=7.8 \mathrm{~Hz}, 2 \mathrm{H}), 7.27$ (t, $J=7.9$ $\mathrm{Hz}, 2 \mathrm{H}), 7.02(\mathrm{t}, J=7.4 \mathrm{~Hz}, 1 \mathrm{H}), 2.07(\mathrm{~s}, 3 \mathrm{H}) \mathrm{ppm} .{ }^{13} \mathbf{C} \mathbf{N M R}\left(101 \mathrm{MHz},\left(\mathrm{CD}_{3}\right)_{2} \mathrm{CO}\right): \delta=$ $168.8(\mathrm{C}), 140.7(\mathrm{C}), 129.5(2 \mathrm{X} \mathrm{CH}), 124.0(\mathrm{CH}$, rotamers), 120.0-119.9 (2 X CH, rotamers), $24.3\left(\mathrm{CH}_{3}\right) \mathrm{ppm}$.

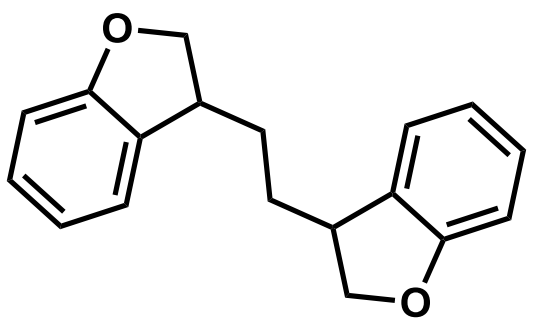

\section{1,2-bis(2,3-dihydrobenzofuran-3-yl)ethane (4u)}

Synthesized according to GP1 affording $5 \mathrm{mg}$ (20\%) of an amorphous solid, isolated as a diasteromeric mixture, characterized according to NMR comparison. ${ }^{13}$

${ }^{1}$ H NMR $\left(400 \mathrm{MHz}, \mathrm{CDCl}_{3}\right) \delta=7.22-7.09(\mathrm{~m}, 4 \mathrm{H}), 6.87$ (tdd, $\left.J=7.4,1.6,1.0 \mathrm{~Hz}, 2 \mathrm{H}\right), 6.83-$ $6.75(\mathrm{~m}, 2 \mathrm{H}), 4.63(\mathrm{t}, J=8.8 \mathrm{~Hz}, 2 \mathrm{H}), 4.22(\mathrm{ddd}, J=8.8,6.2,1.5 \mathrm{~Hz}, 2 \mathrm{H}), 3.52-3.36(\mathrm{~m}, 2 \mathrm{H})$, 1.98-1.85 (m, $1 \mathrm{H}), 1.85-1.68(\mathrm{~m}, 2 \mathrm{H}), 1.66-1.56(\mathrm{~m}, 1 \mathrm{H}) \mathrm{ppm} .{ }^{13} \mathbf{C}$ NMR $\left(101 \mathrm{MHz}, \mathrm{CDCl}_{3}\right) \delta$ $=159.9(2 \times \mathrm{X} \mathrm{C}), 130.4(\mathrm{C}), 130.3(\mathrm{C}), 128.3(2 \mathrm{X} \mathrm{CH}), 124.3(\mathrm{CH}), 124.2(\mathrm{CH}), 120.4(\mathrm{CH})$, $120.4(\mathrm{CH}), 109.6(2 \mathrm{X} \mathrm{CH}), 76.6\left(\mathrm{CH}_{2}\right), 76.5\left(\mathrm{CH}_{2}\right), 41.9(\mathrm{CH}), 41.9(\mathrm{CH}), 32.3\left(\mathrm{CH}_{2}\right), 32.2$ $\left(\mathrm{CH}_{2}\right)$ ppm. 


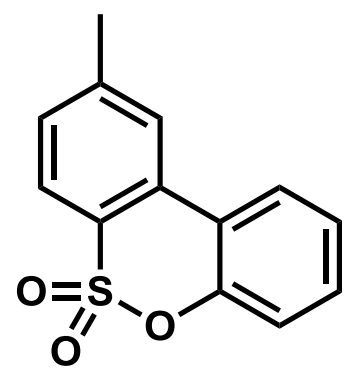

\section{9-methyldibenzo[c,e][1,2] oxathiine 6,6-dioxide (4v)}

Synthesized according to GP1 on a $0.95 \mathrm{mmol}$ scale, affording $131 \mathrm{mg}(56 \%)$ of an amorphous solid, characterized according to NMR comparison. ${ }^{14}$

${ }^{1}$ H NMR $\left(400 \mathrm{MHz}, \mathrm{CDCl}_{3}\right) \delta=7.95(\mathrm{dd}, J=7.8,1.6 \mathrm{~Hz}, 1 \mathrm{H}), 7.90(\mathrm{~d}, J=7.8 \mathrm{~Hz}, 1 \mathrm{H}), 7.76$ (s, $1 \mathrm{H}), 7.48(\mathrm{td}, J=7.8,1.6 \mathrm{~Hz}, 1 \mathrm{H}), 7.43-7.37(\mathrm{~m}, 2 \mathrm{H}), 7.35(\mathrm{dd}, J=8.0,1.2 \mathrm{~Hz}, 1 \mathrm{H}), 2.55$ $(\mathrm{s}, 3 \mathrm{H}) \mathrm{ppm} .{ }^{13} \mathrm{C}$ NMR $\left(101 \mathrm{MHz}, \mathrm{CDCl}_{3}\right) \delta=149.9(\mathrm{C}), 144.6(\mathrm{C}), 131.5(\mathrm{C}), 131.1(\mathrm{CH})$, 129.8 (CH), $129.7(\mathrm{C}), 126.5(\mathrm{CH}), 125.2(\mathrm{CH}), 125.1(\mathrm{CH}), 124.2(\mathrm{CH}), 121.6(\mathrm{C}), 120.0(\mathrm{CH})$, $22.0\left(\mathrm{CH}_{3}\right) \mathrm{ppm}$.
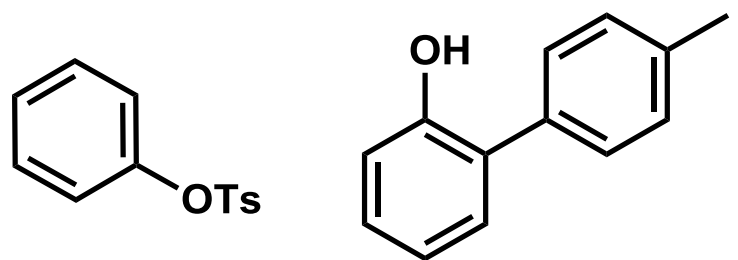

phenyl 4-methylbenzenesulfonate (3v)/4'-methyl-[1,1'-biphenyl]-2-ol (5v) (72:28)

Synthesized according to GP1 on a $0.95 \mathrm{mmol}$ scale, affording $54 \mathrm{mg}(25 \%)$ of an amorphous solid, characterized according to NMR comparison. ${ }^{15}$

3v: ${ }^{1}$ H NMR $\left(400 \mathrm{MHz}, \mathrm{CDCl}_{3}\right) \delta=7.69(\mathrm{~d}, J=8.3 \mathrm{~Hz}, 2 \mathrm{H}), 7.30-7.20(\mathrm{~m}, 5 \mathrm{H}), 7.00-6.93(\mathrm{~m}$, $2 \mathrm{H}), 2.43$ (s, $3 \mathrm{H}) \mathrm{ppm} .{ }^{13} \mathbf{C ~ N M R}\left(101 \mathrm{MHz}, \mathrm{CDCl}_{3}\right) \delta=149.6(\mathrm{C}), 145.3(\mathrm{C}), 132.4(\mathrm{C}), 129.7$ $(2 \mathrm{X} \mathrm{CH}), 129.6(2 \mathrm{X} \mathrm{CH}), 128.5(2 \mathrm{X} \mathrm{CH}), 127.0(\mathrm{CH}), 122.3(2 \mathrm{X} \mathrm{CH}), 21.7\left(\mathrm{CH}_{3}\right) \mathrm{ppm}$.

5v: ${ }^{1}$ H NMR $\left(400 \mathrm{MHz}, \mathrm{CDCl}_{3}\right) \delta=7.35(\mathrm{~d}, J=8.0 \mathrm{~Hz}, 2 \mathrm{H}), 7.30-7.20(\mathrm{~m}, 4 \mathrm{H}), 7.00-6.93(\mathrm{~m}$, $2 \mathrm{H}), 5.25$ (br. s., $1 \mathrm{H}), 2.40$ (s, $3 \mathrm{H}) \mathrm{ppm} .{ }^{13} \mathbf{C}$ NMR $\left(101 \mathrm{MHz}, \mathrm{CDCl}_{3}\right) \delta=152.5(\mathrm{C}), 137.6$ $(\mathrm{C}), 134.0(\mathrm{C}), 130.2(2 \mathrm{X} \mathrm{CH}), 129.9(2 \mathrm{X} \mathrm{CH}), 128.9(2 \mathrm{X} \mathrm{CH}), 128.0(\mathrm{C}), 120.7(\mathrm{CH}), 115.7$ $(\mathrm{CH}), 21.2\left(\mathrm{CH}_{3}\right) \mathrm{ppm}$.

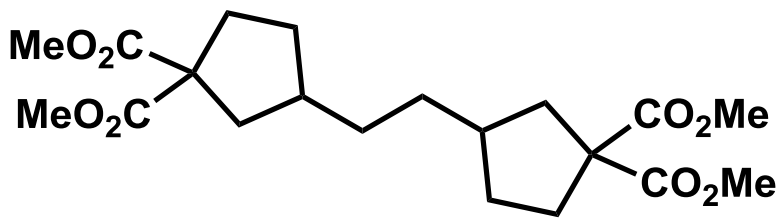

tetramethyl 3,3'-(ethane-1,2-diyl)bis(cyclopentane-1,1-dicarboxylate) (7a)

Synthesized according to GP2 on $0.2 \mathrm{mmol}$ scale, affording $21 \mathrm{mg}(53 \%)$ of an off yellow oil as a mixture of diastereomers.

IR (neat, $\mathbf{~ c m}^{-1}$ ): 2953(m), 2853(m), 1729(vs), 1435(s), 1251(s), 1157(s). ${ }^{1} \mathbf{H}$ NMR (400 MHz, $\left.\mathrm{CDCl}_{3}\right): \delta=3.72(\mathrm{~s}, 6 \mathrm{H}), 3.72(\mathrm{~s}, 6 \mathrm{H}), 2.45(\mathrm{dd}, J=13.1,7.3 \mathrm{~Hz}, 2 \mathrm{H}), 2.35-2.25(\mathrm{~m}, 2 \mathrm{H}), 2.18$ 2.09 (m, $2 \mathrm{H}), 1.95-1.80$ (m, $4 \mathrm{H}), 1.68$ (ddd, $J=13.2,9.9,1.2 \mathrm{~Hz}, 2 \mathrm{H}), 1.35-1.19$ (m, $6 \mathrm{H}$ ) ppm. ${ }^{13} \mathrm{C}$ NMR $\left(101 \mathrm{MHz}, \mathrm{CDCl}_{3}\right) \delta=173.2(4 \mathrm{X} \mathrm{C}), 59.9(2 \mathrm{X} \mathrm{C}), 52.6\left(2 \mathrm{X} \mathrm{CH}_{3}\right), 52.6(2 \mathrm{X}$ $\left.\mathrm{CH}_{3}\right), 40.8\left(2 \mathrm{X} \mathrm{CH}_{2}\right), 39.9(\mathrm{CH}), 39.9(\mathrm{CH}), 34.1\left(\mathrm{CH}_{2}\right), 34.1\left(\mathrm{CH}_{2}\right), 33.9\left(2 \mathrm{X} \mathrm{CH}_{2}\right), 32.1(2 \mathrm{X}$ $\mathrm{CH}_{2}$ ) ppm. HRMS (EI) $\mathrm{m} / \mathrm{z}$ calc'd for $\mathrm{C}_{20} \mathrm{H}_{30} \mathrm{O}_{8}\left[\mathrm{M}^{+}\right]$398.1941, found [M-124] 274.1240. 


\section{1,6-diphenylhexane (7b)}

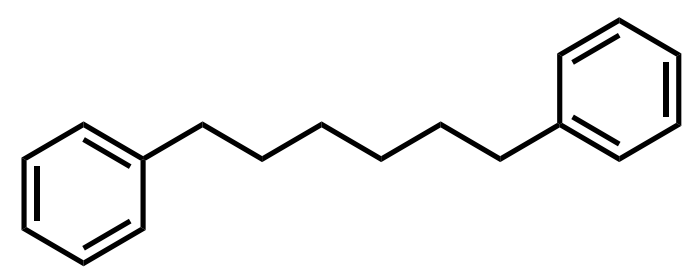

Synthesized according to GP2, affording $23 \mathrm{mg}(50 \%)$ of an off yellow oil, characterized according to NMR comparison. ${ }^{16}$

${ }^{1}$ H NMR (400 MHz, $\left.\mathrm{CDCl}_{3}\right): \delta=7.32-7.27(\mathrm{~m}, 4 \mathrm{H}), 7.22-7.16(\mathrm{~m}, 6 \mathrm{H}), 2.65-2.58(\mathrm{~m}, 4 \mathrm{H})$, 1.69-1.59 (m, $4 \mathrm{H}), 1.44-1.35(\mathrm{~m}, 4 \mathrm{H}) \mathrm{ppm} .{ }^{13} \mathbf{C}$ NMR $\left(101 \mathrm{MHz}, \mathrm{CDCl}_{3}\right): \delta=142.8(2 \mathrm{X} \mathrm{C})$, $128.4(4 \mathrm{X} \mathrm{CH}), 128.2(4 \mathrm{X} \mathrm{CH}), 125.6(2 \mathrm{X} \mathrm{CH}), 35.9\left(2 \mathrm{X} \mathrm{CH}_{2}\right), 31.4\left(2 \mathrm{X} \mathrm{CH}_{2}\right), 29.1(2 \mathrm{X}$ $\left.\mathrm{CH}_{2}\right) \mathrm{ppm}$.

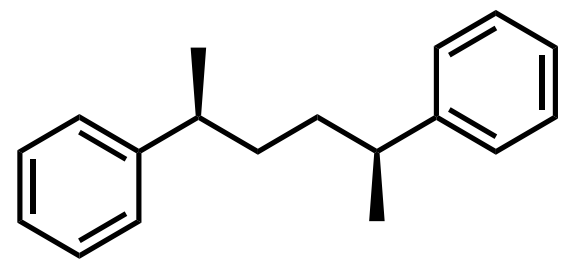

((2S,5S)-hexane-2,5-diyl)dibenzene (7c)

Synthesized according to GP2 (51\%) using $(\boldsymbol{R})$-(1-bromopropan-2-yl)benzene (6c) that was synthesized according to Barriault`s method ${ }^{17}$, affording $24 \mathrm{mg}(51 \%)$ of an off yellow oil, characterized according to NMR comparison. ${ }^{18}$

${ }^{1} \mathbf{H}$ NMR $\left(400 \mathrm{MHz}, \mathrm{CDCl}_{3}\right): \delta=7.31-7.26(\mathrm{~m}, 4 \mathrm{H}), 7.21-7.11(\mathrm{~m}, 6 \mathrm{H}), 2.72-2.60(\mathrm{~m}, 2 \mathrm{H})$, $1.54-1.44(\mathrm{~m}, 4 \mathrm{H}), 1.21(\mathrm{~d}, J=6.9 \mathrm{~Hz}, 6 \mathrm{H}) \mathrm{ppm} .{ }^{13} \mathbf{C} \mathbf{N M R}\left(101 \mathrm{MHz}, \mathrm{CDCl}_{3}\right): \delta=147.6(2 \mathrm{X}$ C), $128.2(4 \mathrm{X} \mathrm{CH}), 127.0(4 \mathrm{X} \mathrm{CH}), 125.8(2 \mathrm{X} \mathrm{CH}), 40.0(2 \mathrm{X} \mathrm{CH}), 36.1\left(2 \mathrm{X} \mathrm{CH}_{2}\right), 22.5(2 \mathrm{X}$ $\left.\mathrm{CH}_{3}\right) \mathrm{ppm}$.

$[\boldsymbol{\alpha}]_{\mathbf{D}}{ }^{23}=+13.0^{\circ}\left(\mathrm{c} 1.0, \mathrm{CHCl}_{3}\right)$.

Corresponding racemic $\mathbf{6 c}$ analog provided a statistical mixture of diastereomers under GP2 in similar yield and appearance, characterized according to NMR comparison.

${ }^{1}$ H NMR (400 MHz, $\left.\mathrm{CDCl}_{3}\right): \delta=7.32-7.24(\mathrm{~m}, 4 \mathrm{H}), 7.21-7.09(\mathrm{~m}, 6 \mathrm{H}), 2.70-2.57(\mathrm{~m}, 2 \mathrm{H})$, $1.61-1.39(\mathrm{~m}, 4 \mathrm{H}), 1.20(\mathrm{~d}, J=7.0 \mathrm{~Hz}, 6 \mathrm{H}) \mathrm{ppm} .{ }^{13} \mathbf{C} \mathbf{N M R}\left(101 \mathrm{MHz}, \mathrm{CDCl}_{3}\right): \delta=147.7(2 \mathrm{X}$ C), $147.6(2 \mathrm{X} \mathrm{C}), 128.3$ ( $4 \mathrm{X} \mathrm{CH}), 128.2$ ( $4 \mathrm{X} \mathrm{CH}), 127.0(4 \mathrm{X} \mathrm{CH}), 127.0(4 \mathrm{X} \mathrm{CH}), 125.8(2 \mathrm{X}$ $\mathrm{CH}$, both diastereomers), $40.1(2 \mathrm{X} \mathrm{CH}), 40.0(2 \mathrm{X} \mathrm{CH}), 36.5\left(2 \mathrm{X} \mathrm{CH}_{2}\right), 36.1\left(2 \mathrm{X} \mathrm{CH}_{2}\right), 22.5(2$ $\left.\mathrm{X} \mathrm{CH}_{3}\right), 22.3\left(2 \mathrm{X} \mathrm{CH}_{3}\right)$ ppm. 


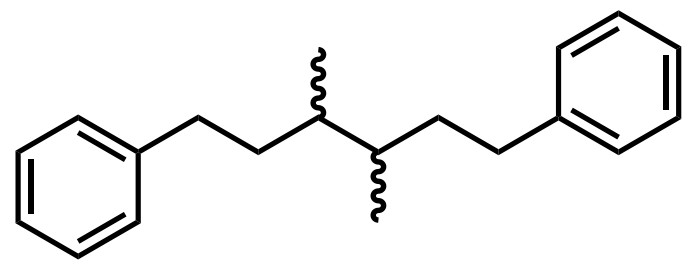

(3,4-dimethylhexane-1,6-diyl)dibenzene (7d)

Synthesized according to GP2 $0.258 \mathrm{mmol}$ scale, affording $18 \mathrm{mg}(51 \%)$ of an off yellow oil as a statistical diastereomeric mixture, characterized according to NMR comparison. ${ }^{19}$

${ }^{1}$ H NMR $\left(400 \mathrm{MHz}, \mathrm{CDCl}_{3}\right): \delta=7.32-7.27(\mathrm{~m}, 4 \mathrm{H}), 7.22-7.15(\mathrm{~m}, 6 \mathrm{H}), 2.72-2.43(\mathrm{~m}, 4 \mathrm{H})$, $1.71-1.60$ (m, $2 \mathrm{H}), 1.54-1.32$ (m, $4 \mathrm{H}), 0.93(\mathrm{~d}, J=6.7 \mathrm{~Hz}, 3 \mathrm{H}), 0.87(\mathrm{~d}, J=6.5 \mathrm{~Hz}, 3 \mathrm{H}) \mathrm{ppm}$. ${ }^{13}$ C NMR (101 MHz, $\left.\mathrm{CDCl}_{3}\right): \delta=143.1(2 \mathrm{X} \mathrm{C}), 143.1(2 \mathrm{X} \mathrm{C}), 128.4(4 \mathrm{X} \mathrm{CH}), 128.3(4 \mathrm{X}$ $\mathrm{CH}), 128.3(4 \mathrm{X} \mathrm{CH}), 128.3(4 \mathrm{X} \mathrm{CH}), 125.6(2 \mathrm{X} \mathrm{CH}), 125.6(2 \mathrm{X} \mathrm{CH}), 37.1(2 \mathrm{X} \mathrm{CH}), 36.9(2$ $\left.\mathrm{X} \mathrm{CH}_{2}\right), 36.2(2 \mathrm{X} \mathrm{CH}), 35.0\left(2 \mathrm{X} \mathrm{CH}_{2}\right), 34.1\left(2 \mathrm{X} \mathrm{CH}_{2}\right), 34.0\left(2 \mathrm{X} \mathrm{CH}_{2}\right), 16.3\left(2 \mathrm{X} \mathrm{CH}_{3}\right), 14.3$ $\left(2 \mathrm{X} \mathrm{CH}_{3}\right) \mathrm{ppm}$.

\section{bibenzyl (7e)}

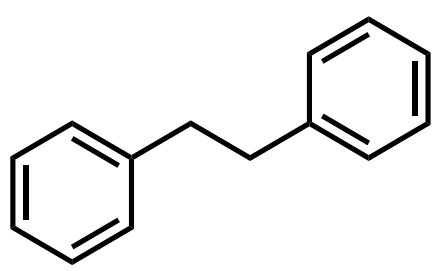

Synthesized according to GP2, affording $28 \mathrm{mg}(77 \%)$ of an off yellow oil, characterized according to NMR comparison. ${ }^{20}$

${ }^{1}$ H NMR (400 MHz, $\left.\mathrm{CDCl}_{3}\right): \delta=7.35-7.28(\mathrm{~m}, 4 \mathrm{H}), 7.26-7.19(\mathrm{~m}, 6 \mathrm{H}), 2.96(\mathrm{~s}, 4 \mathrm{H}) \mathrm{ppm}$.

${ }^{13}$ C NMR (101 MHz, $\left.\mathrm{CDCl}_{3}\right): \delta=141.8(2 \mathrm{X} \mathrm{C}), 128.4(4 \mathrm{X} \mathrm{CH}), 128.3(4 \mathrm{X} \mathrm{CH}), 125.9(2 \mathrm{X}$ $\mathrm{CH}), 37.9\left(2 \mathrm{X} \mathrm{CH}_{2}\right) \mathrm{ppm}$.

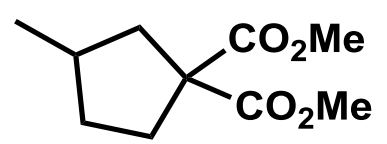

dimethyl 3-methylcyclopentane-1,1-dicarboxylate (8a)

Synthesized according to GP2 on $0.2 \mathrm{mmol}$ scale with addition of 1,4-cyclohexadiene (0.6 mmol, 3 equiv.), affording $27 \mathrm{mg}$ (68\%) of an off yellow oil, characterized according to NMR comparison. ${ }^{21}$

${ }^{1}$ H NMR $\left(400 \mathrm{MHz}, \mathrm{CDCl}_{3}\right) \delta=3.72(\mathrm{~s}, 6 \mathrm{H}), 2.46(\mathrm{dd}, J=13.4,7.3 \mathrm{~Hz}, 1 \mathrm{H}), 2.39-2.28(\mathrm{~m}$, $1 \mathrm{H}), 2.16$ (ddd, $J=13.6,9.3,7.6 \mathrm{~Hz}, 1 \mathrm{H}), 2.09-1.99(\mathrm{~m}, 1 \mathrm{H}), 1.91-1.80(\mathrm{~m}, 1 \mathrm{H}), 1.68(\mathrm{dd}, J=$ 13.3, $10.2 \mathrm{~Hz}, 1 \mathrm{H}), 1.31-1.20(\mathrm{~m}, 1 \mathrm{H}), 1.02(\mathrm{~d}, J=6.5 \mathrm{~Hz}, 3 \mathrm{H}) \mathrm{ppm} .{ }^{13} \mathbf{C}$ NMR $(101 \mathrm{MHz}$, $\left.\mathrm{CDCl}_{3}\right) \delta=173.3(\mathrm{C}), 60.2(\mathrm{C}), 52.6\left(\mathrm{CH}_{3}\right), 52.6\left(\mathrm{CH}_{3}\right), 42.7\left(\mathrm{CH}_{2}\right), 34.4(\mathrm{CH}), 34.2\left(\mathrm{CH}_{2}\right), 34.1$ $\left(\mathrm{CH}_{2}\right), 19.5\left(\mathrm{CH}_{3}\right) \mathrm{ppm}$. 


\section{References}

1. Chen, Y.-A.; Liu, C.-Y. RSC Adv. 2015, 5, 74180.

2. Xu, Z.; Xiao, Y.; Ding, H.; Cao, C.; Li, H.; Pang, G.; Shi, Y. Synthesis 2015, 47, 1560.

3. Vogler, T.; Studer, A. Adv. Synth. Catal. 2008, 350, 1963.

4. Buter, J.; Heijnen, D.; Vila, C.; Hornillos, V.; Otten, E.; Giannerini, M.; Minnaard, A. J.;

Feringa, B. L. Angew. Chem. Int. Ed. 2016, 55, 3620.

5. Kirai, N.; Yamamoto, Y. Eur. J. Org. Chem. 2009, 1864.

6. Puthiaraj, P.; Suresh, P.; Pitchumani, K. Green Chem. 2014, 16, 2865.

7. Zhou, Q.; Wang, Y.-N.; Guo, X.-Q.; Zhu, X.-H.; Li, Z.-M.; Hou, X.-F. Organometallics 2015, 34,1021 .

8. Xiong, Q.; Fu, Z.; Li, Z.; Cai, H. Synlett 2015, 26, 975.

9. Nishimura, N.; Yoza, K.; Kobayashi, K. J. Am. Chem. Soc. 2010, 132, 777.

10. Wang, L.; Murai, Y.; Yoshida, T.; Ishida, A.; Masuda, K.; Sakihama, Y.; Hashidoko, Y.;

Hatanaka, Y.; Hashimoto, M. Org. Lett. 2015, 17, 616.

11. van der Born, D.; Sewing, C.; Herscheid, J. D. M.; Windhorst, A. D.; Orru, R. V. A.; Vugts, D. J. Angew. Chem. Int. Ed. 2014, 53, 11046.

12. (a) Camilleri, P.; Kirby, A. J.; Lewis, R. J.; Sanders, J. K. M. J. Chem. Soc., Chem. Commun. 1988, 1537. (b) Penner, G. H.; Wasylishen, R. E. Can. J. Chem. 1989, 67, 525.

13. Lhermet, R.; Durandetti, M.; Maddaluno, J. Beilstein J. Org. Chem. 2013, 9, 710.

14. Revol, G.; McCallum, T.; Morin, M.; Gagosz, F.; Barriault, L. Angew. Chem. Int. Ed. 2013, 52,13342 .

15. (a) Miller, S. C. J. Org. Chem. 2010, 75, 4632. (b) Yang, K.; Zhang, J.; Li, Y.; Cheng, B.; Zhao, L.; Zhai, H. Org. Lett. 2013, 15, 808.

16. Horino, Y.; Takahashi, Y.; Koketsu, K.; Abe, H.; Tsuge, K. Org. Lett. 2014, 16, 3184.

17. McTiernan, C. D.; Morin, M.; McCallum, T.; Scaiano, J. C.; Barriault, L. Catal. Sci.

Technol. 2016, 6, 201.

18. (a) Bywater, S.; Lachance, P.; Worsfold, D. J. J. Phys. Chem. 1975, 79, 2148. (b) Dulog, L.;

David, K.-H. Makromol. Chem. 1976, 177, 1717.

19. Ozaki, S.; Matsushita, H.; Ohmori, H. J. Chem. Soc., Perkin Trans. 1. 1993, 649.

20. Zhu, J.; Pérez, M.; Caputo, C. B.; Stephan, D. W. Angew. Chem. Int. Ed. 2016, 55, 1417.

21. Millán, A.; Álvarez de Cienfuegos, L.; Miguel, D.; Campaña, A. G.; Cuerva, J. M. Org. Lett.

2012, 14, 5984. 
5. Spectra of New Compounds

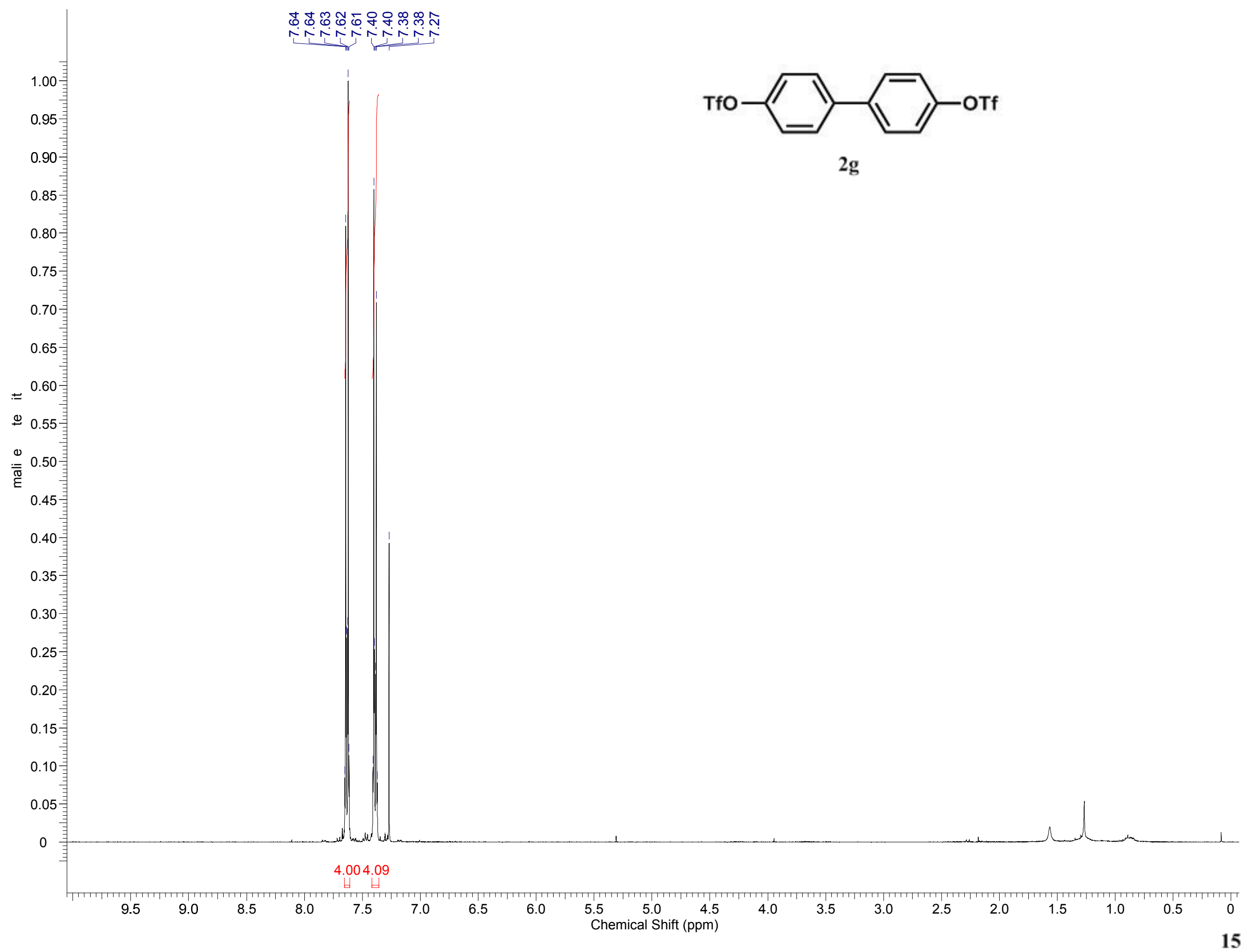




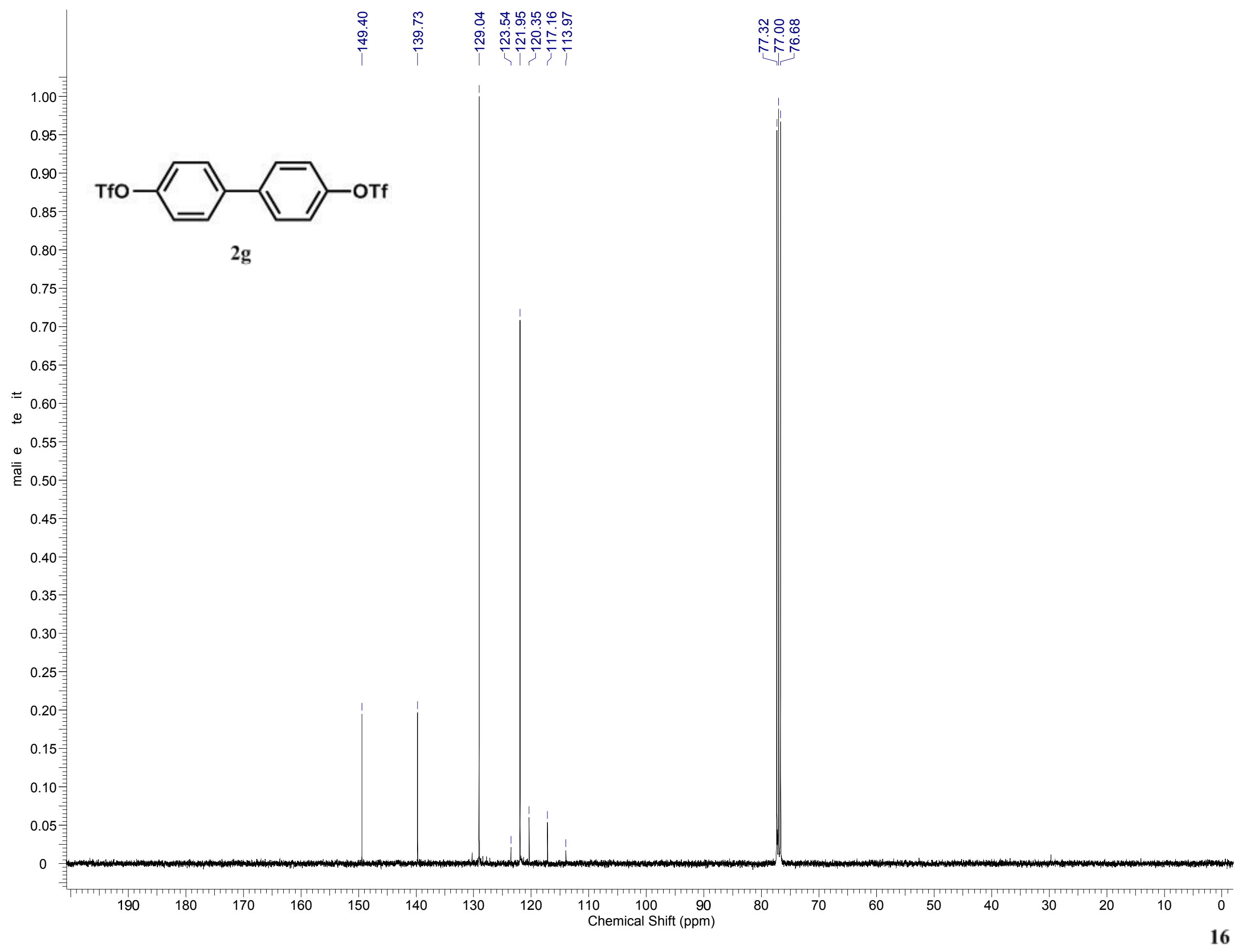




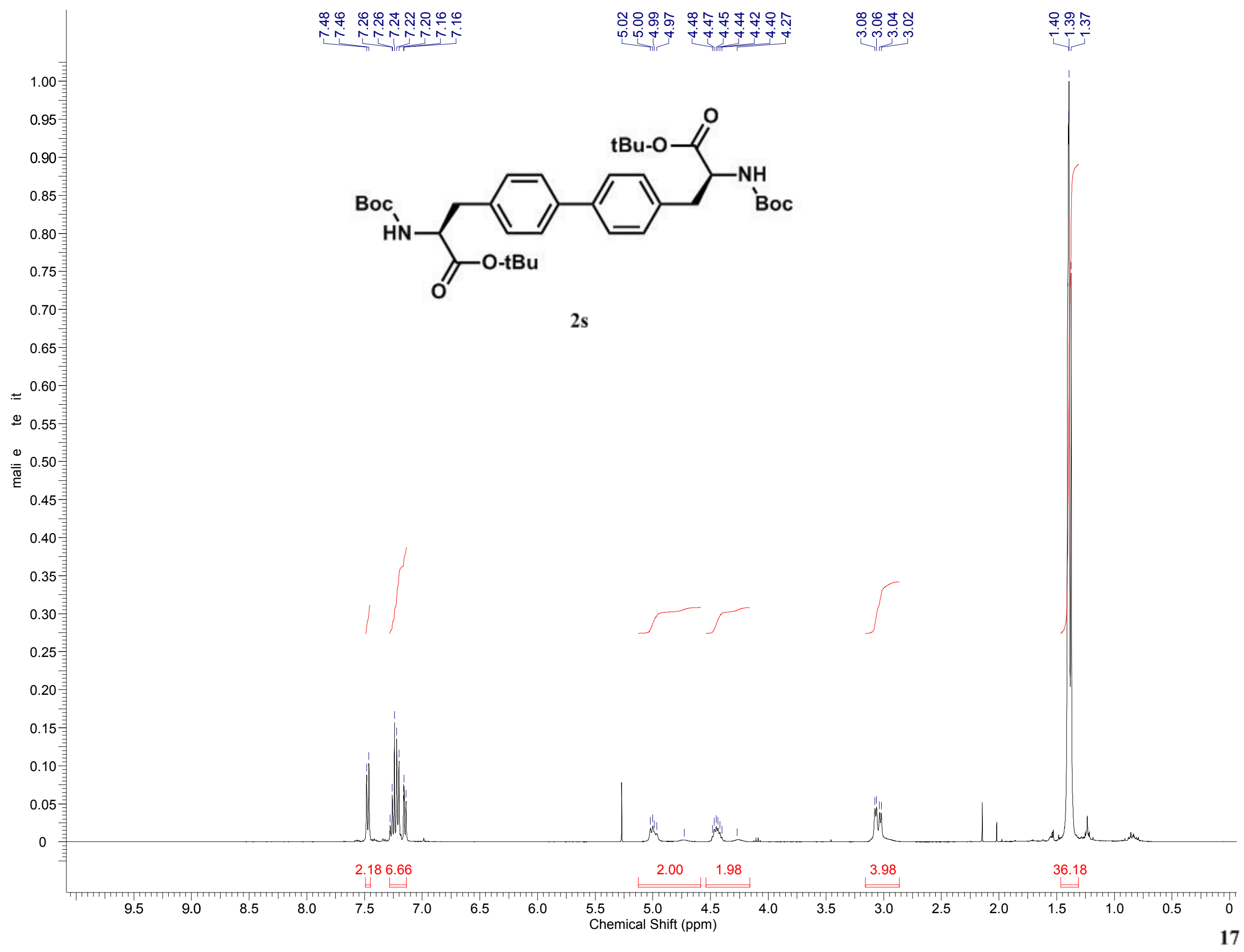




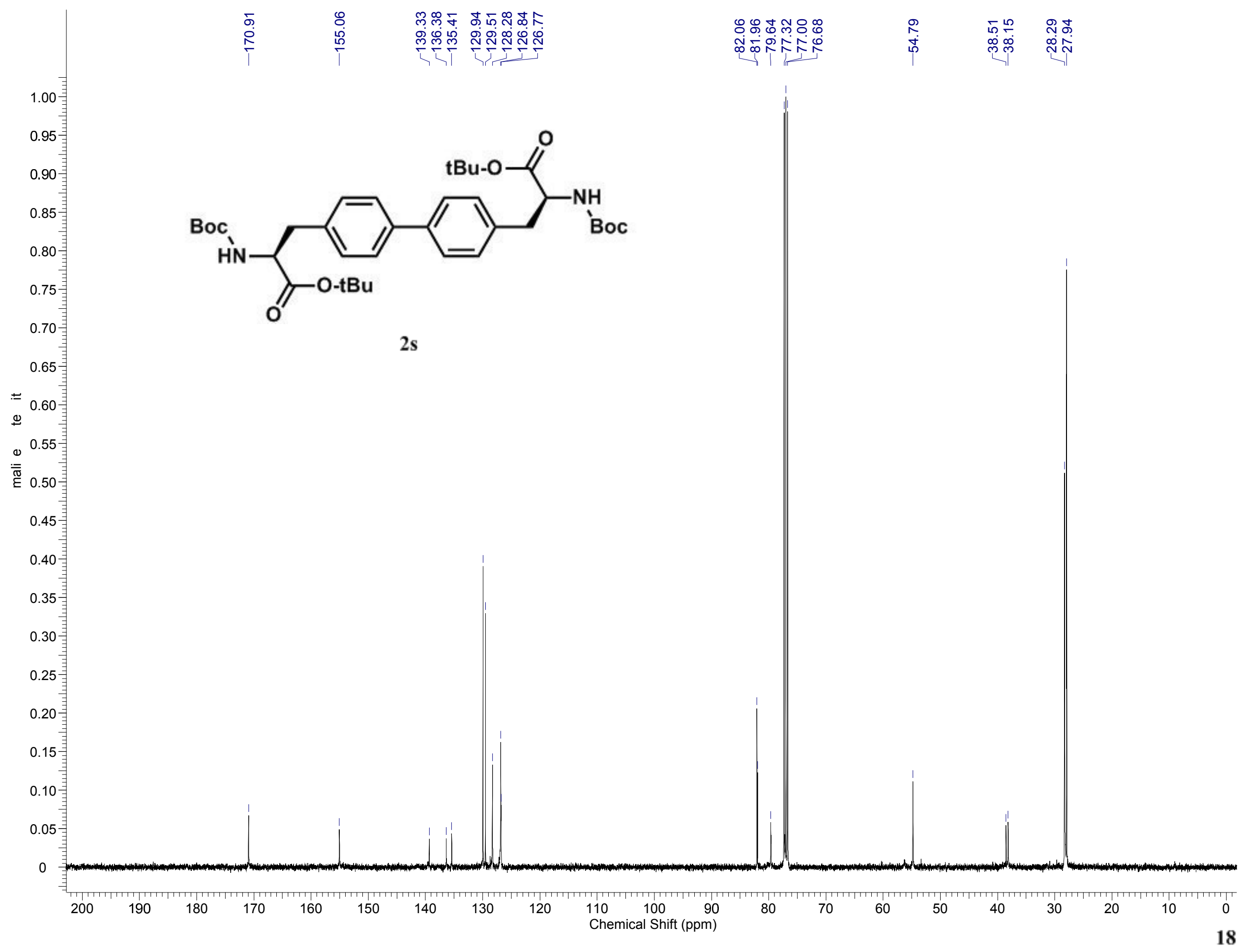




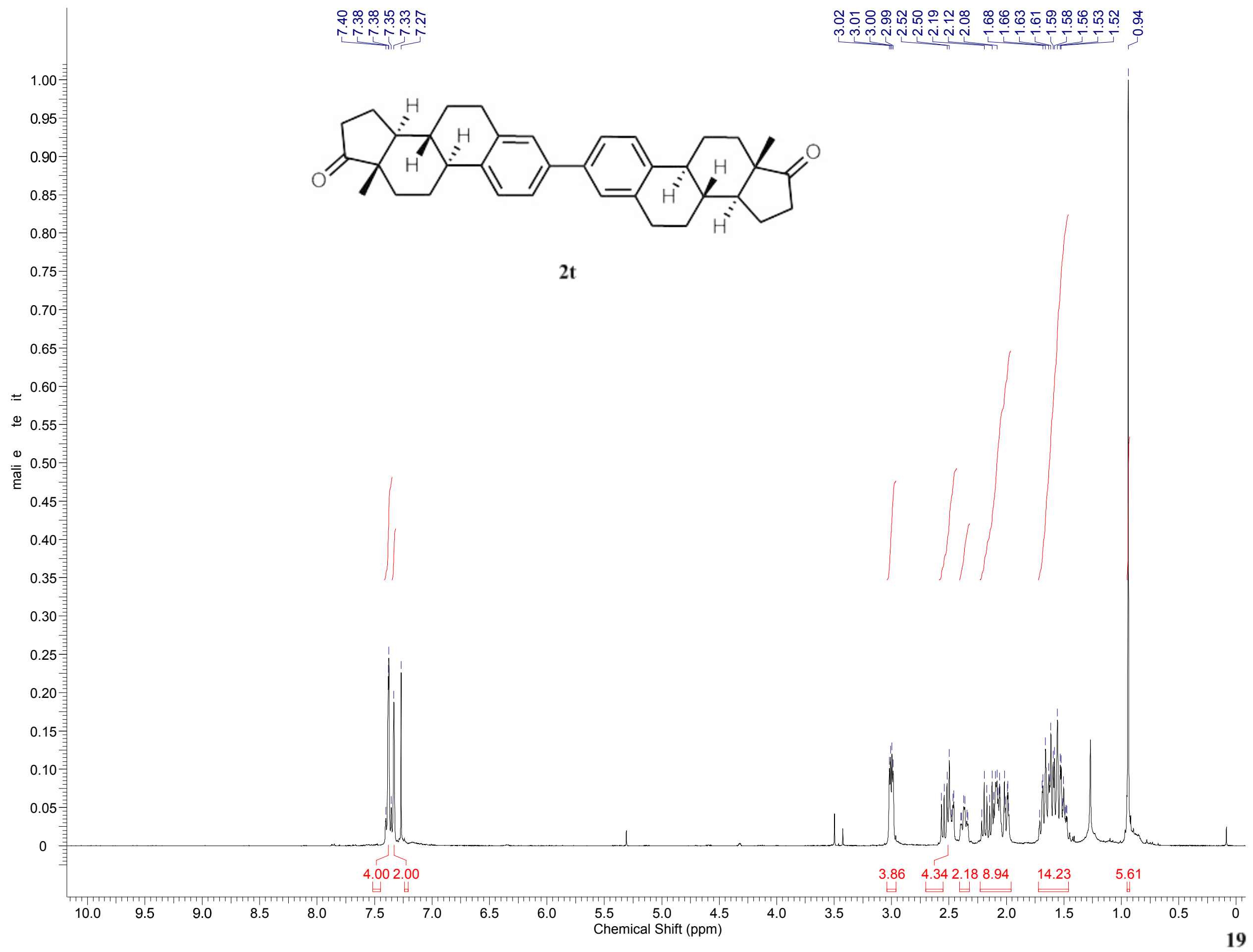




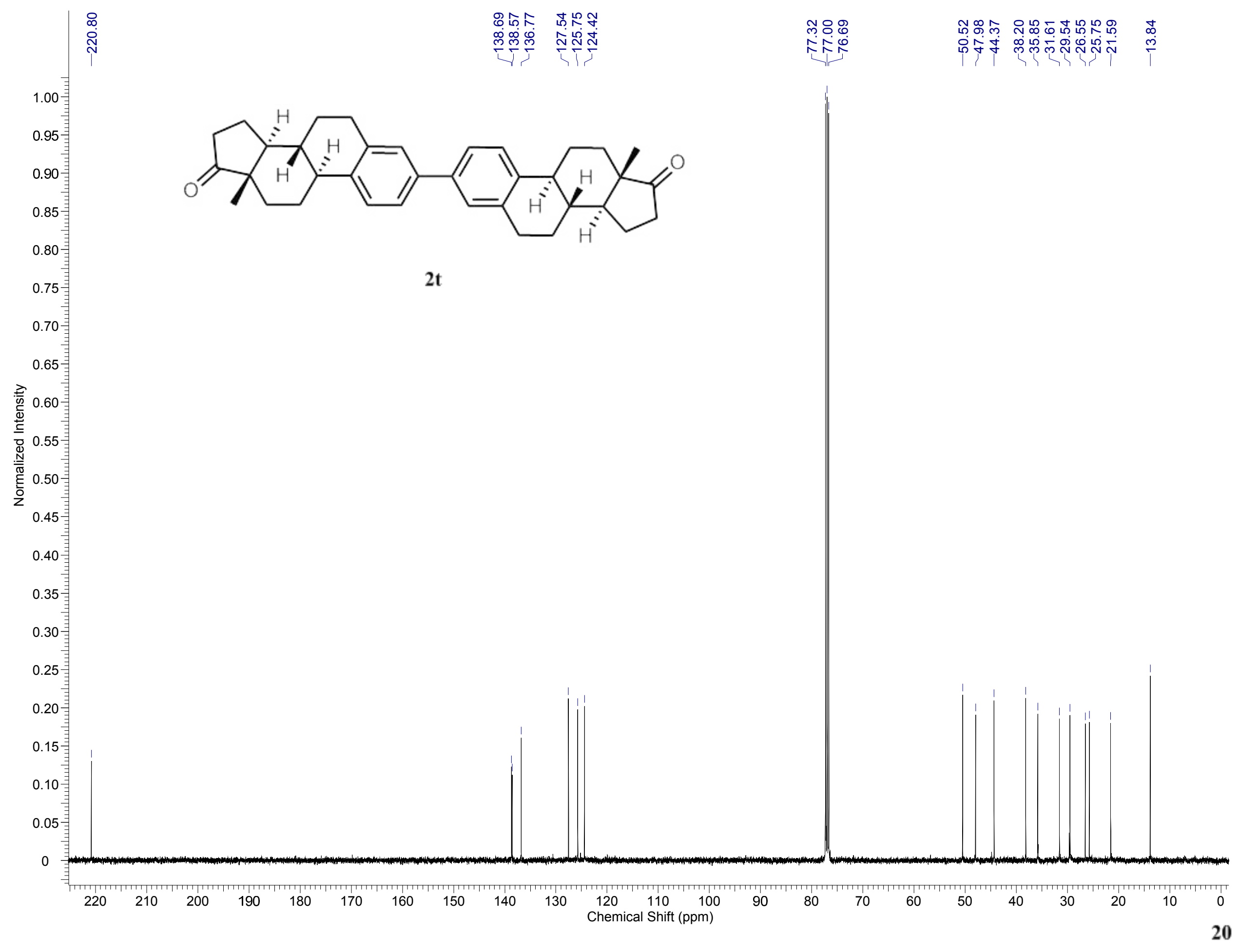




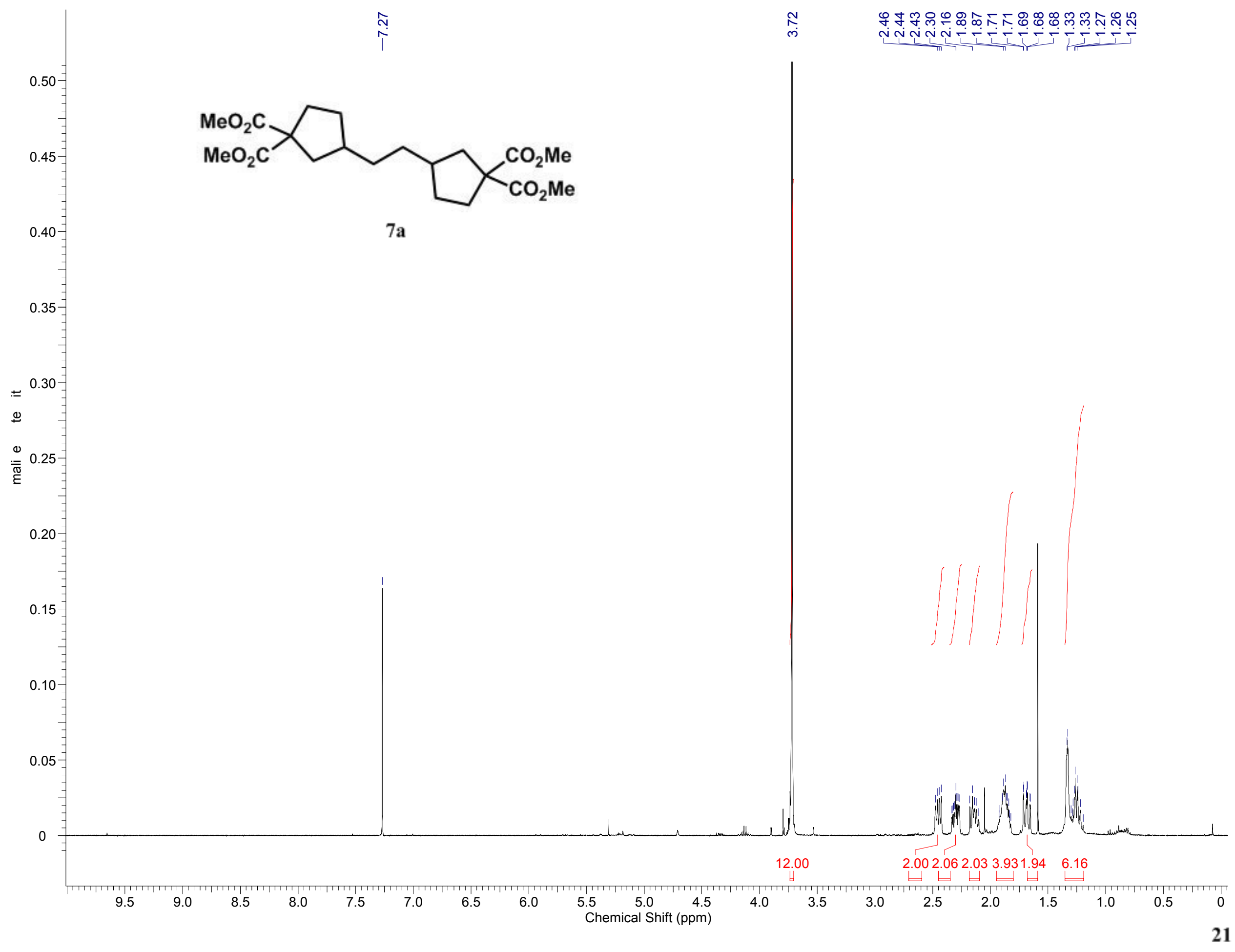




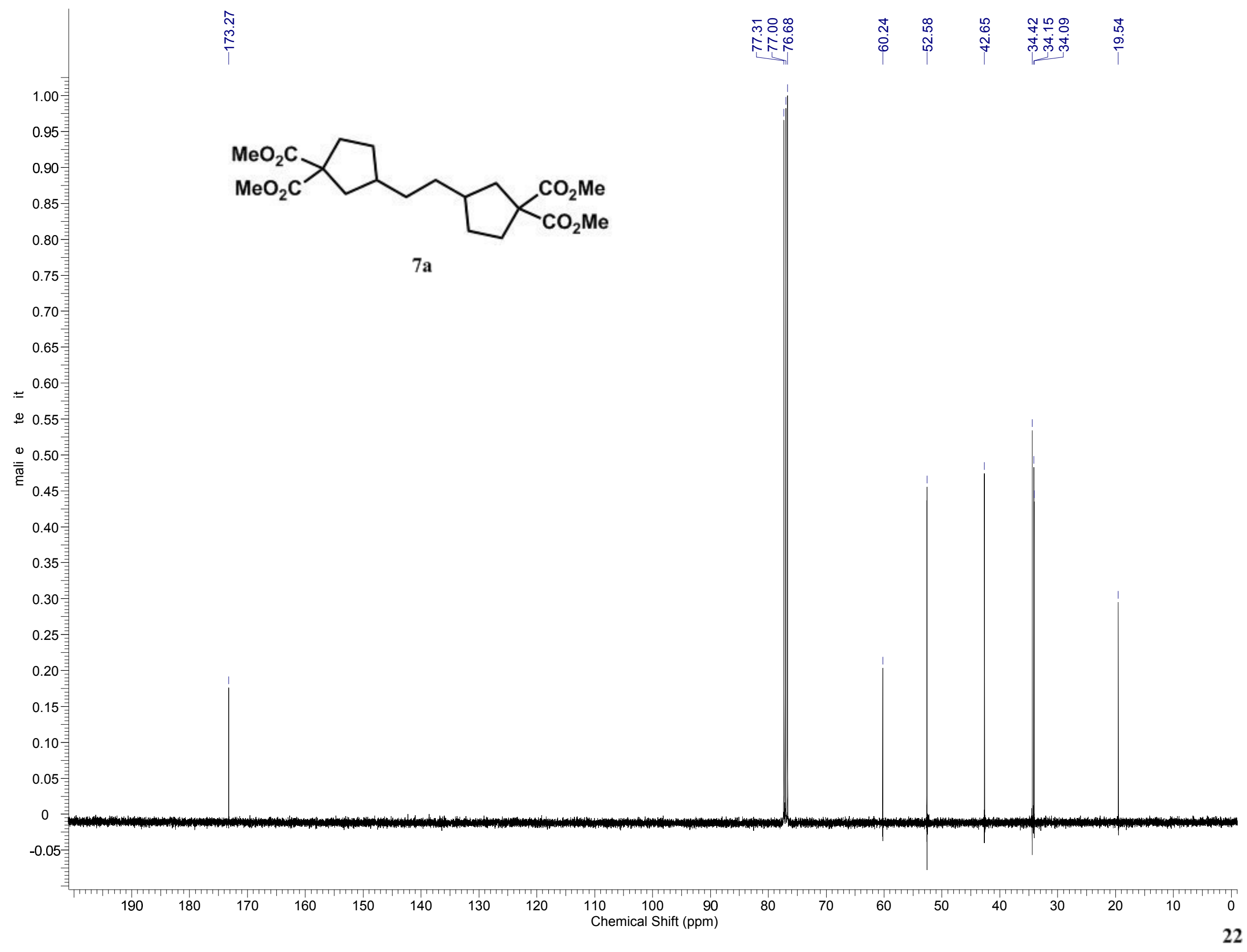




\section{1H NMR Spectra of Known Compounds}

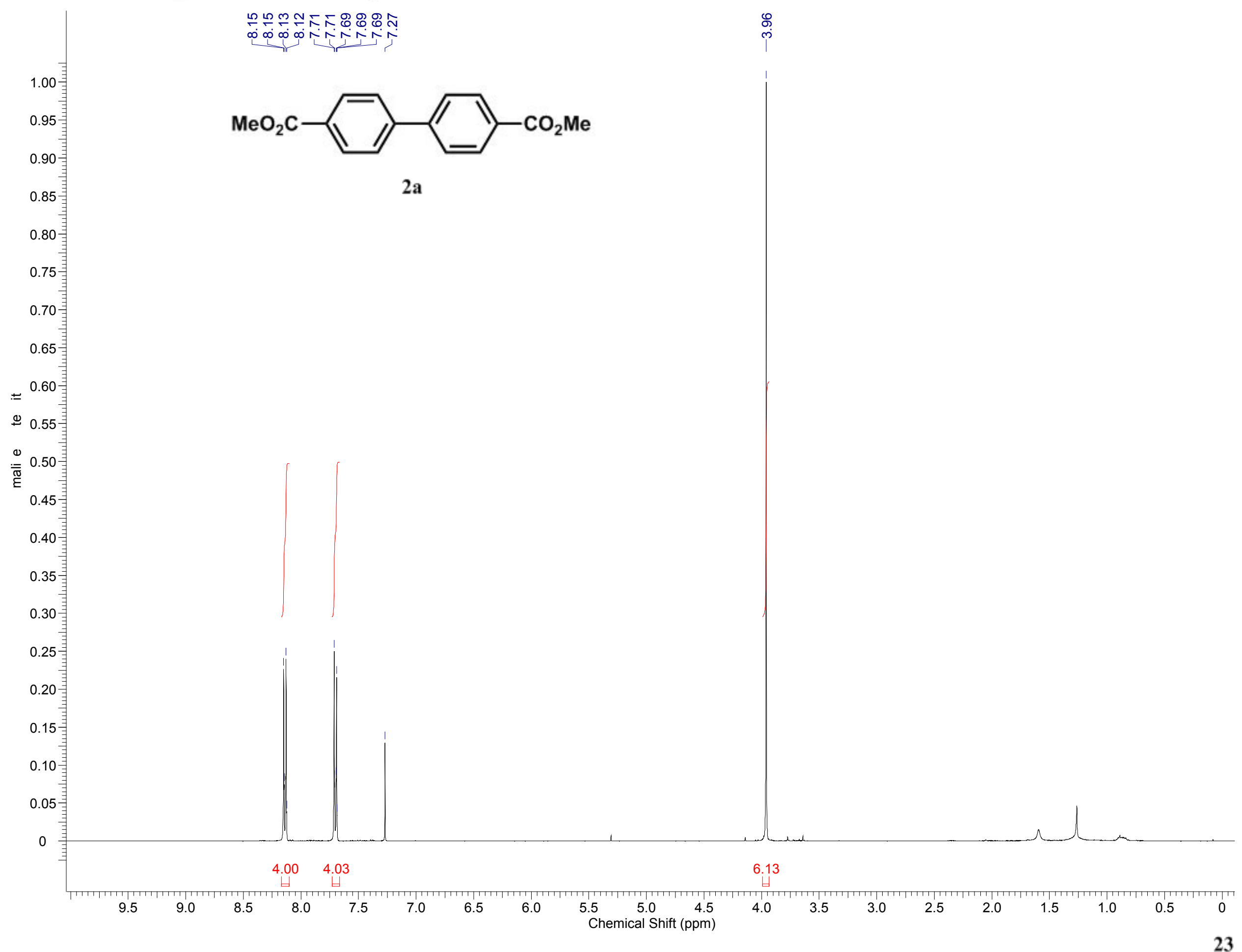




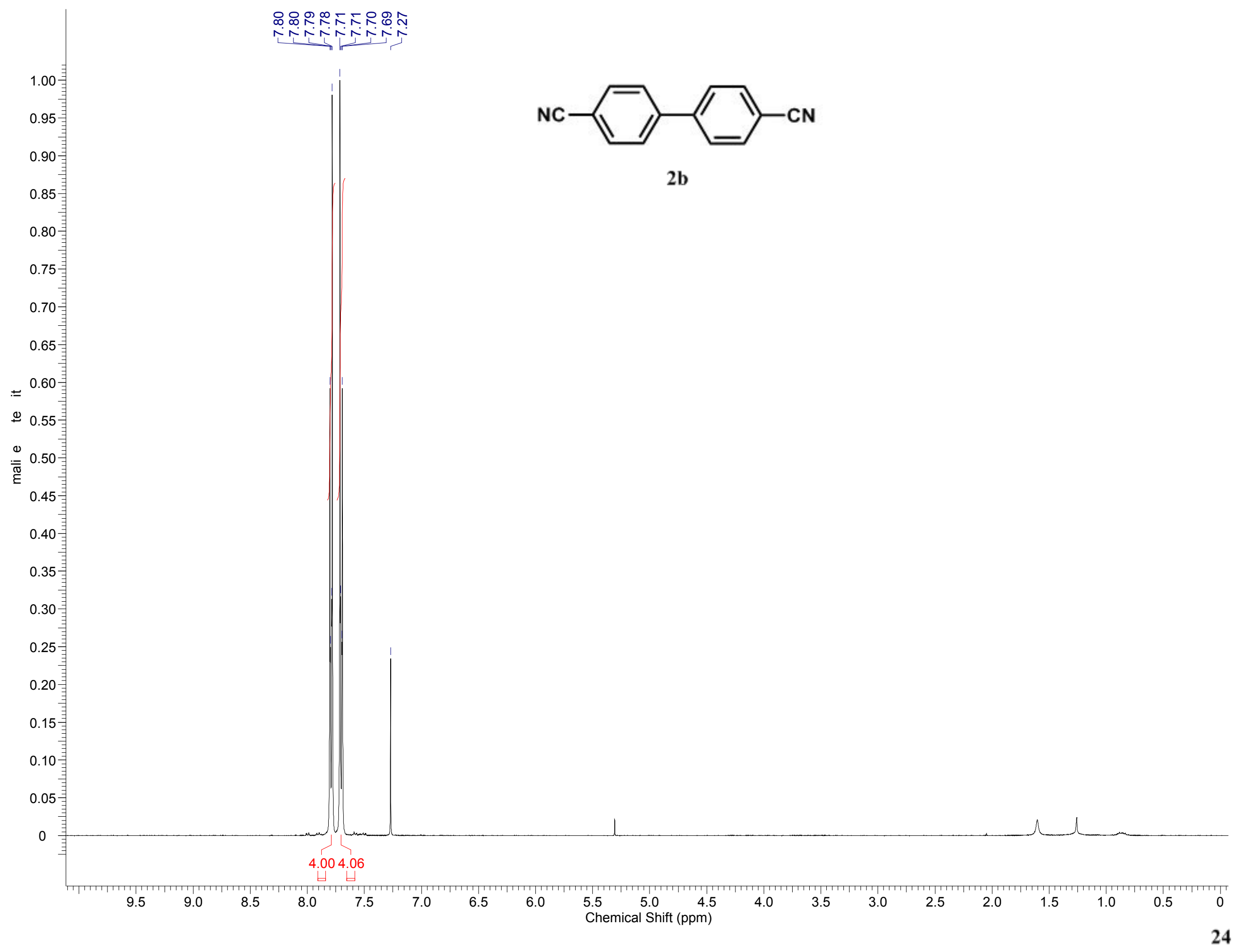




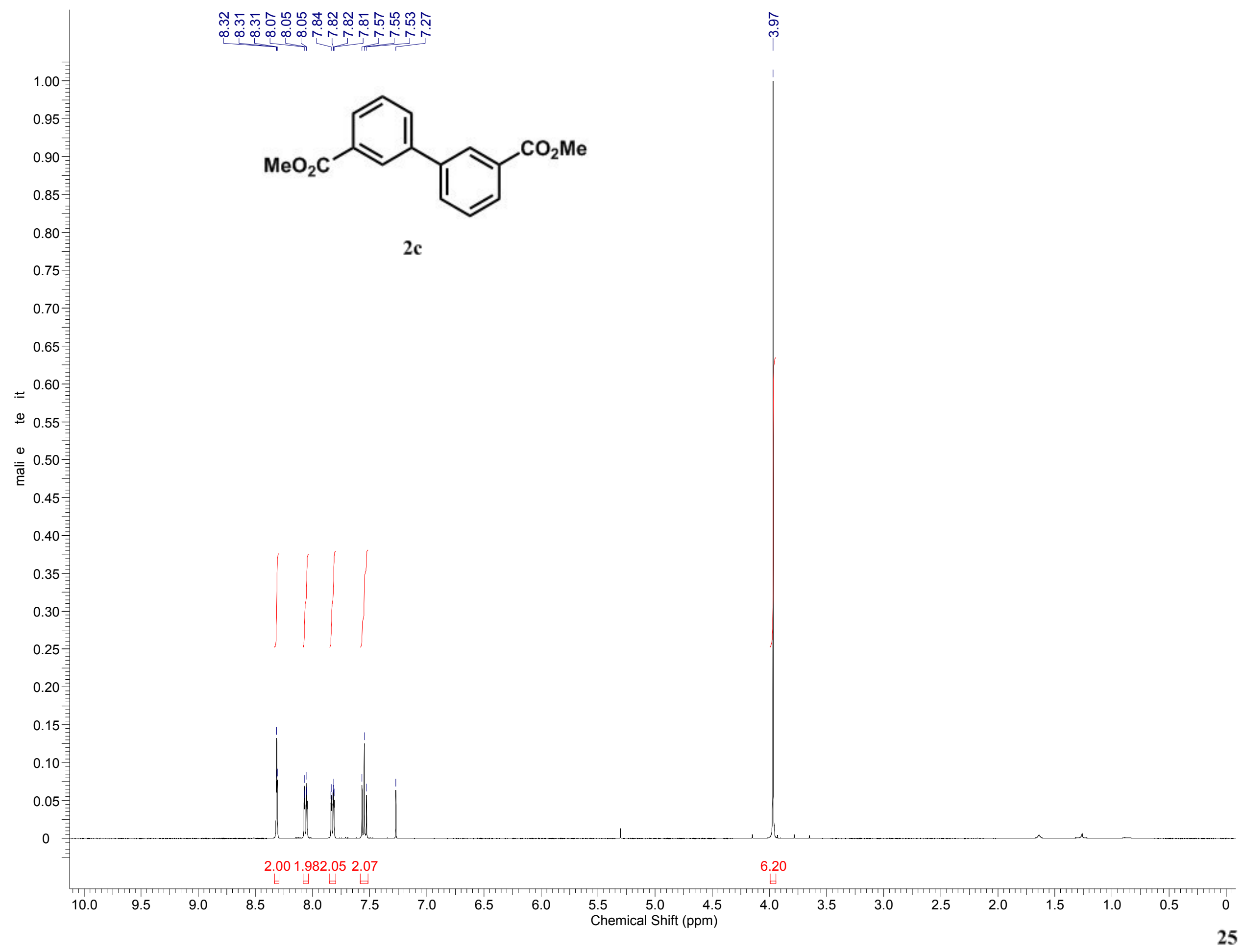




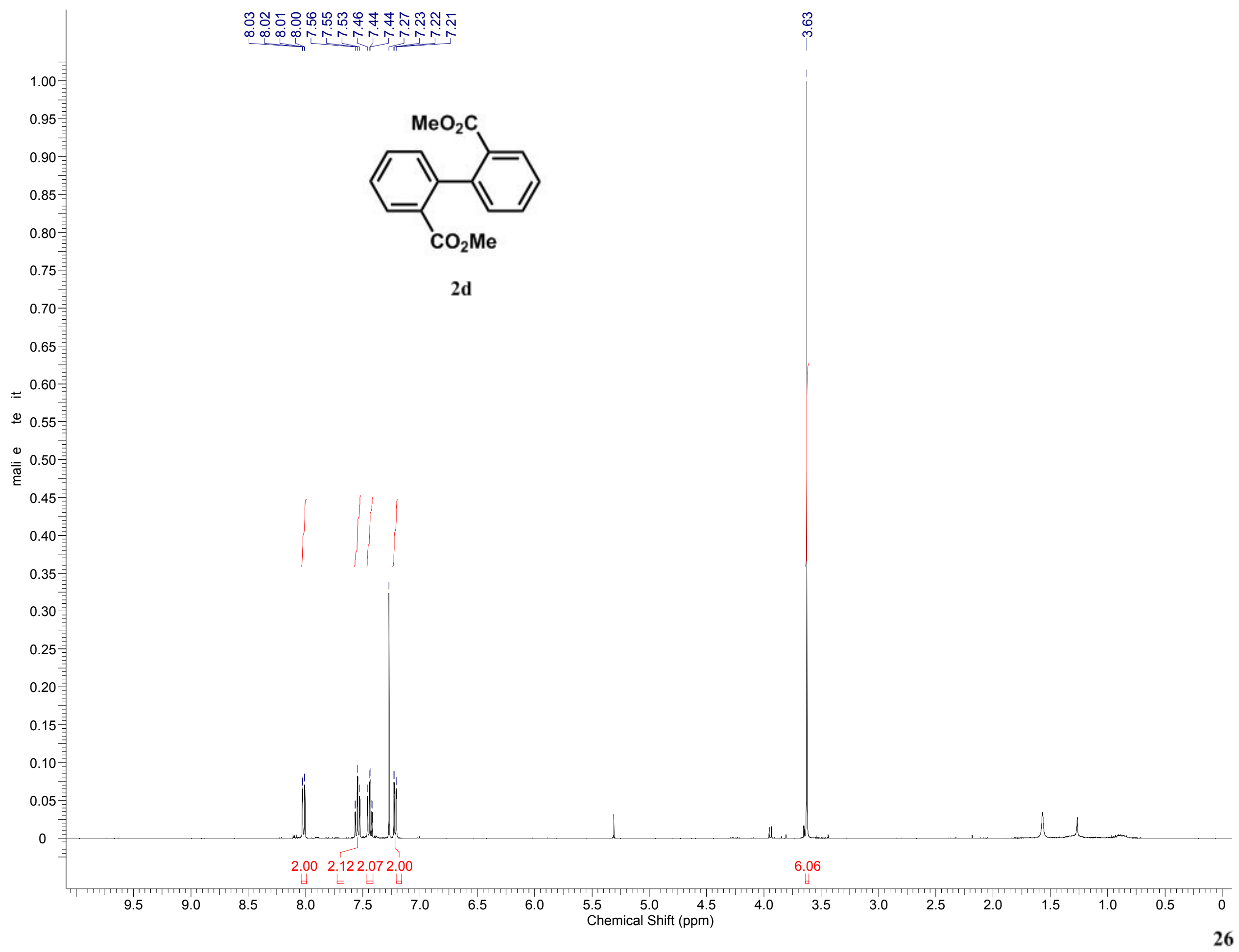




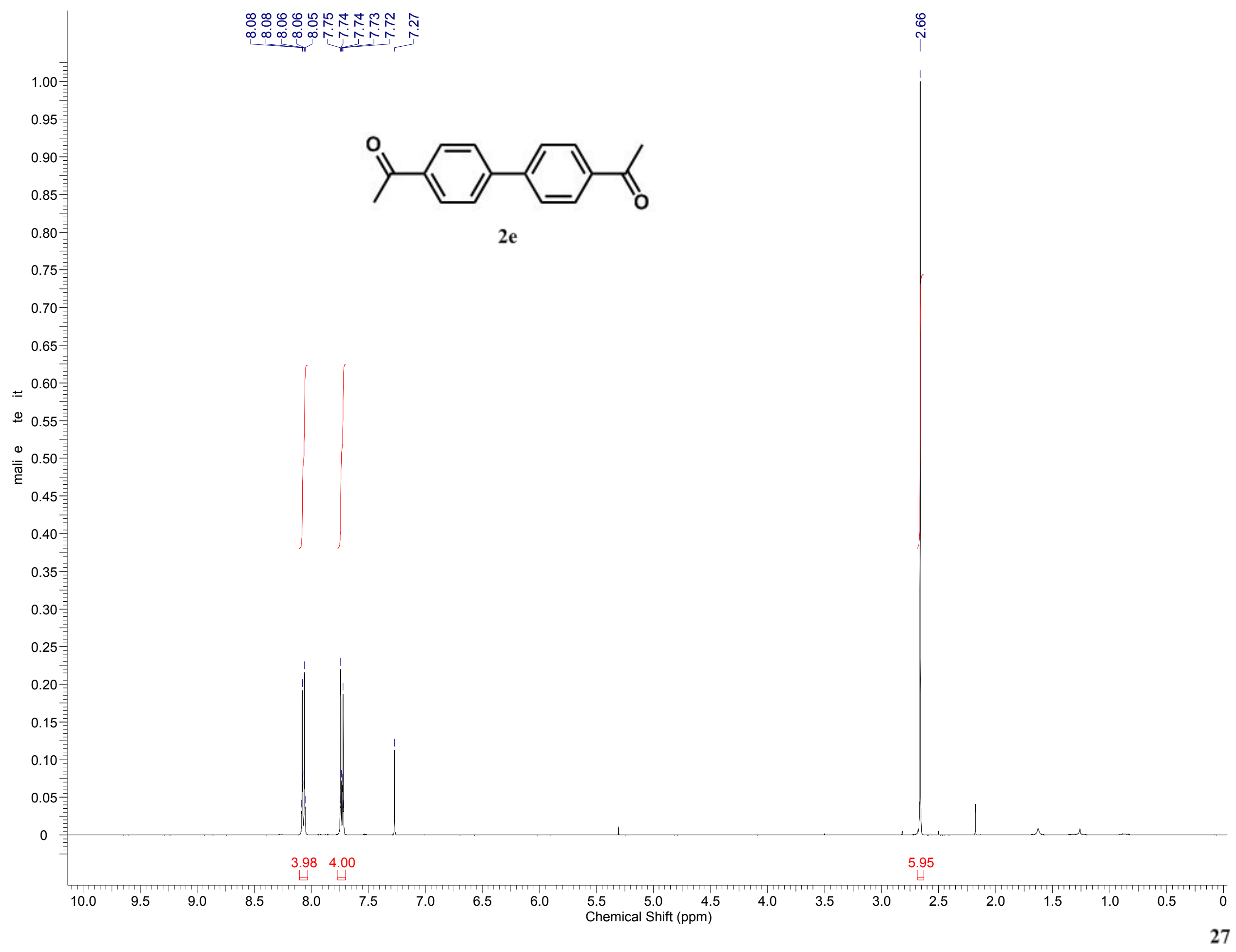




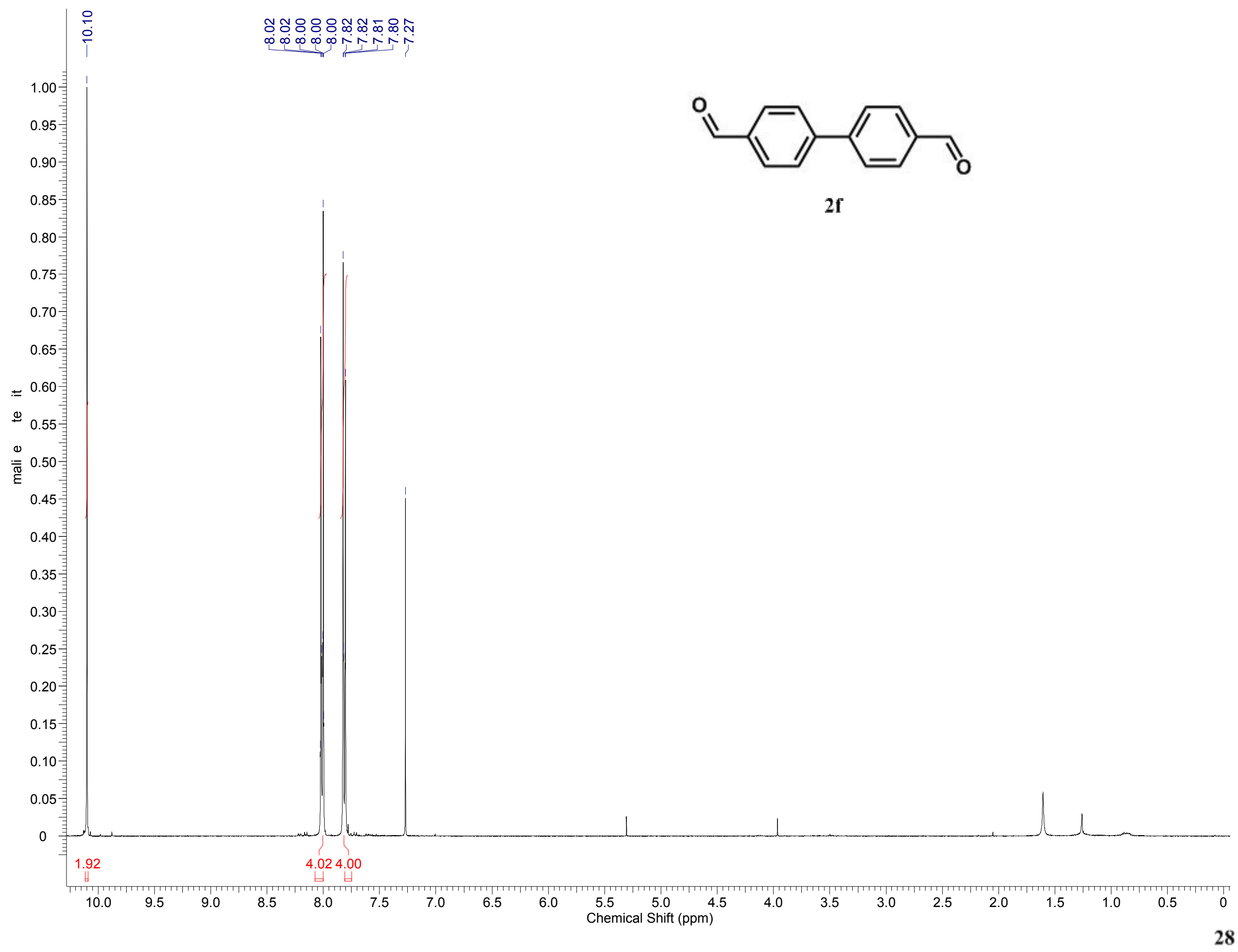




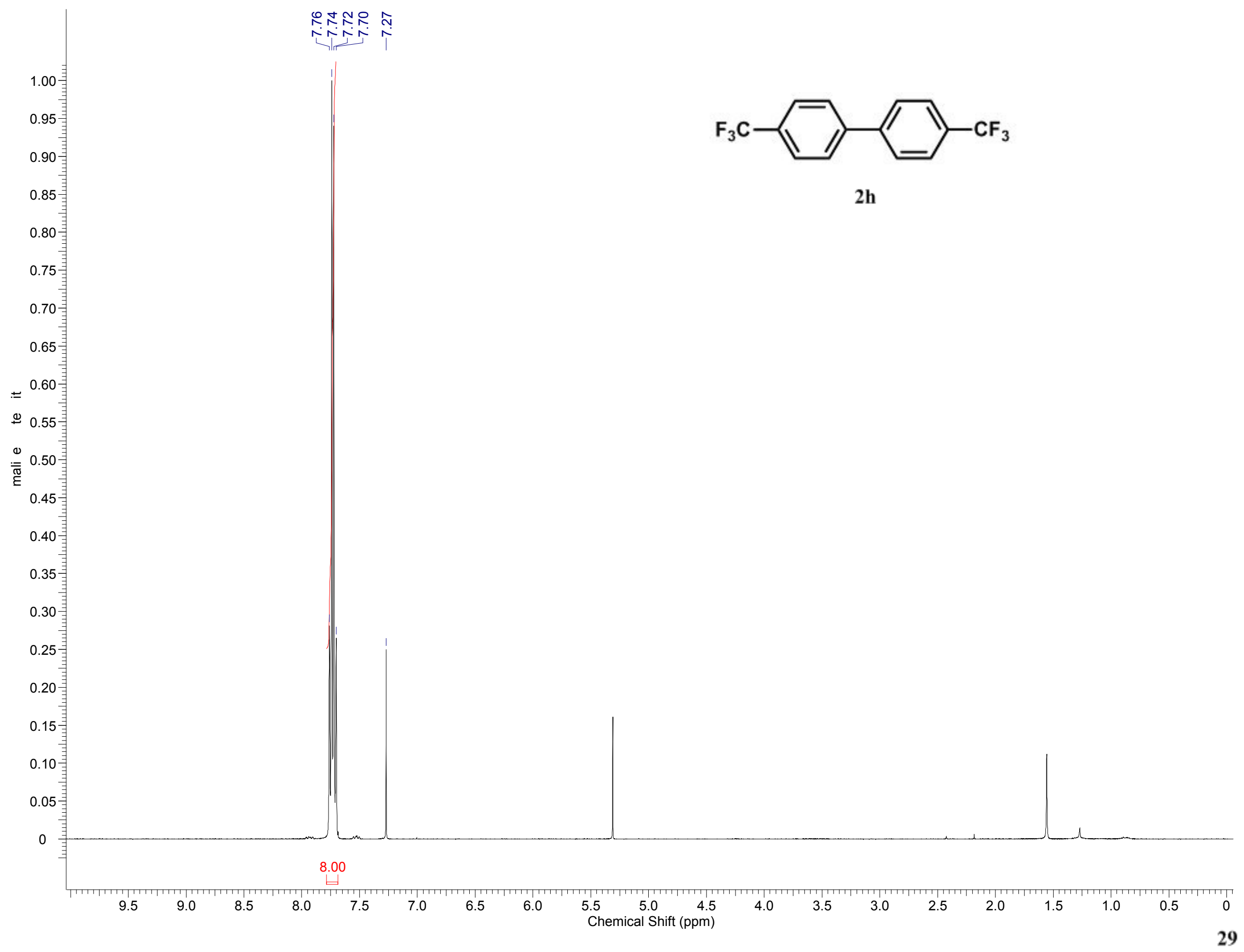




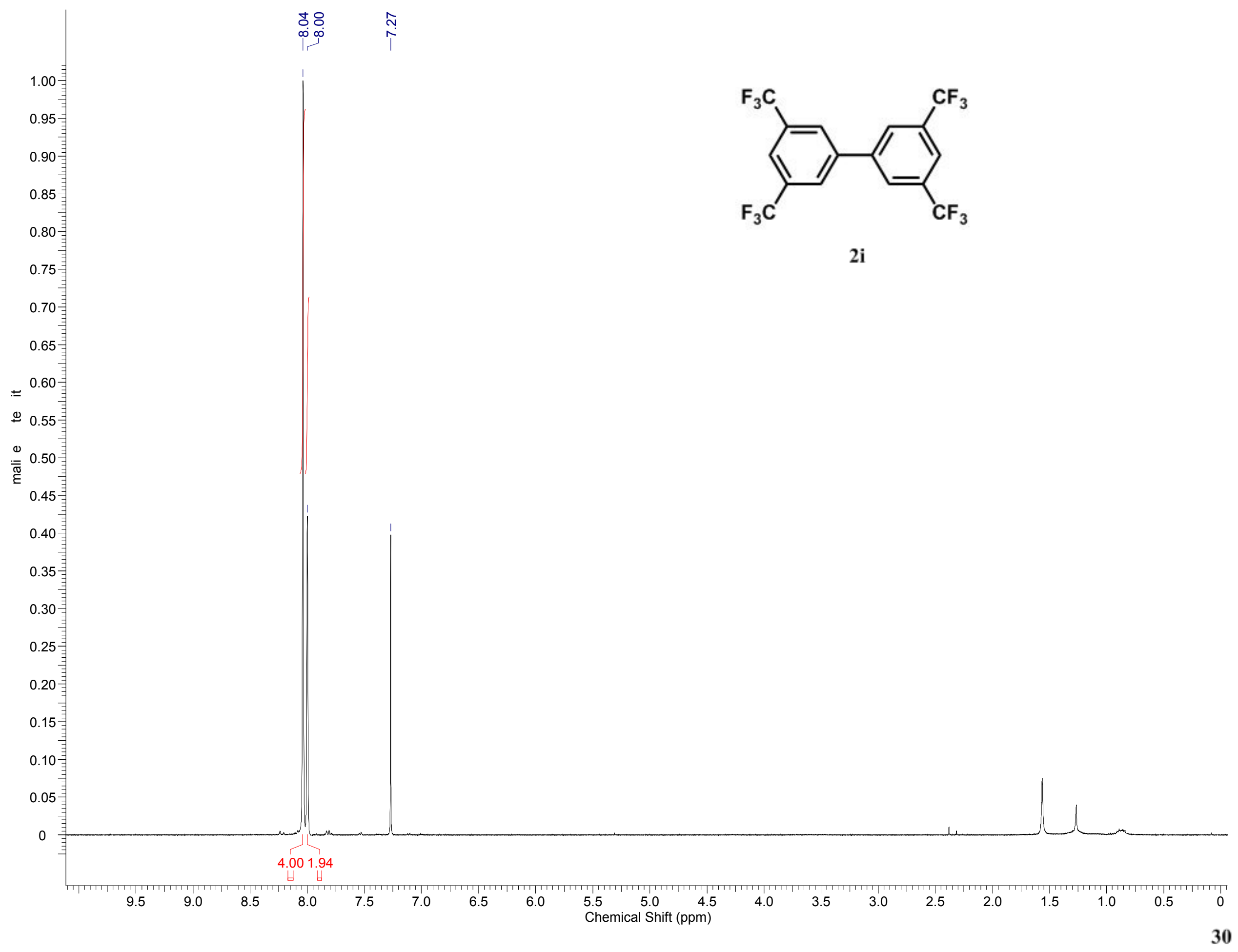




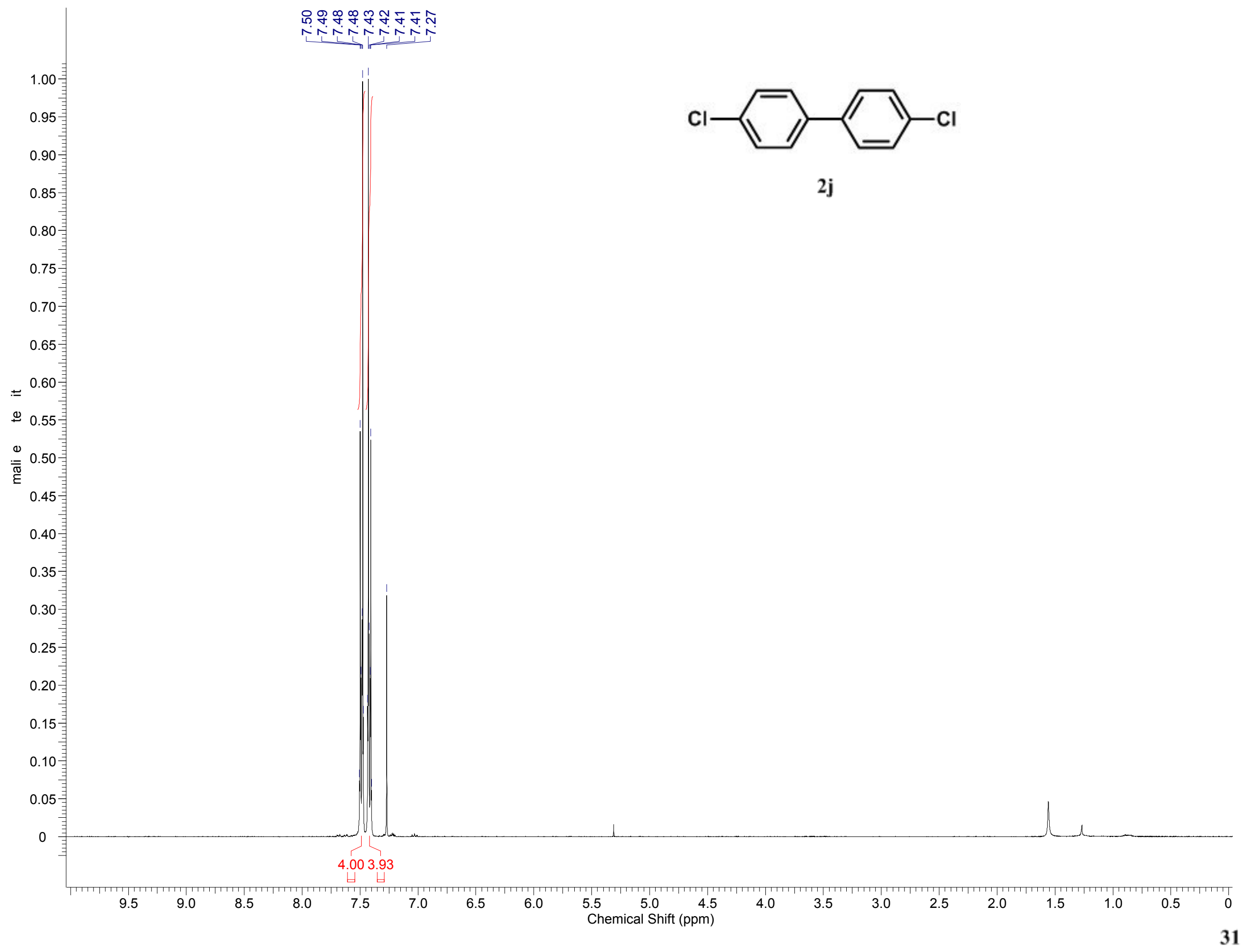




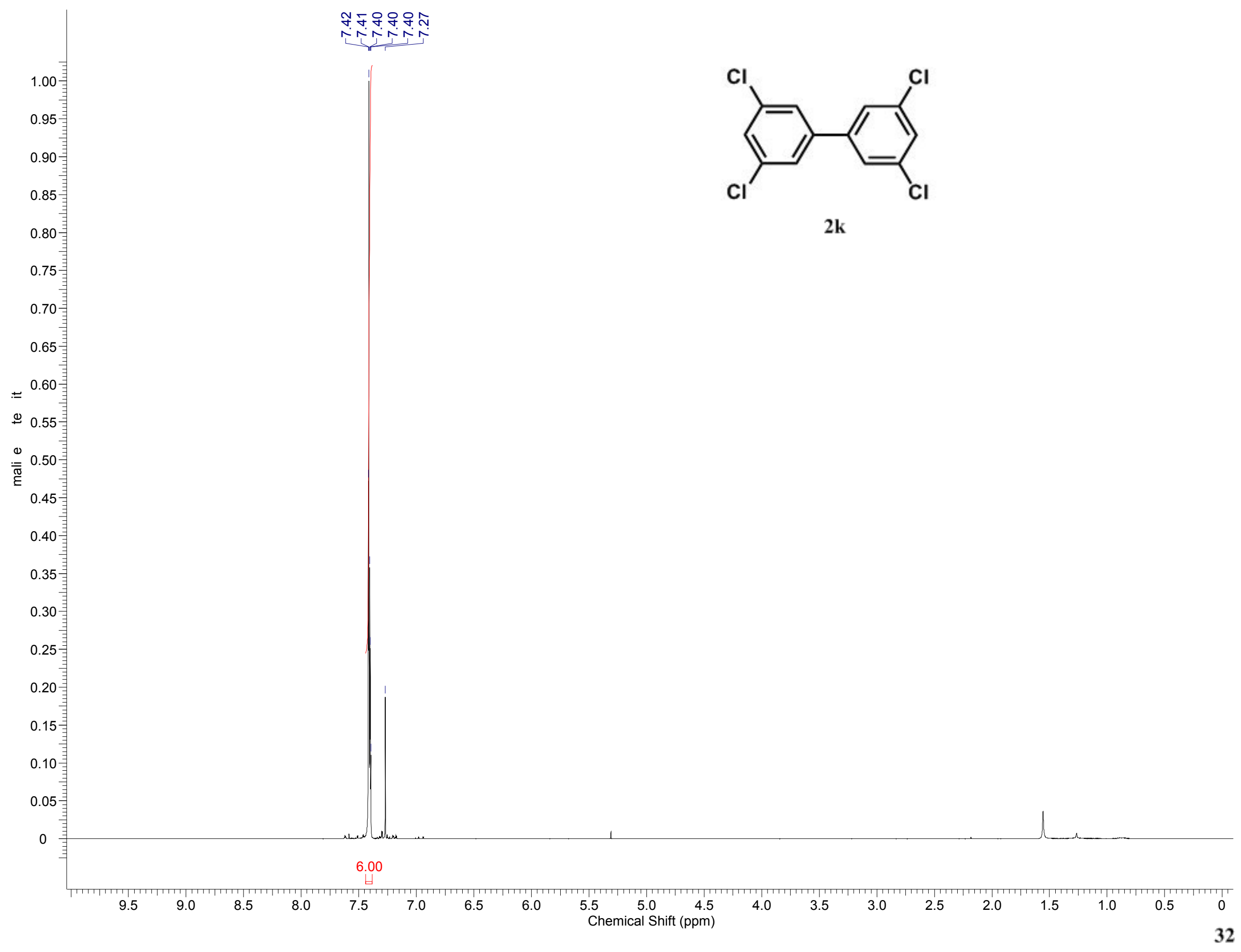




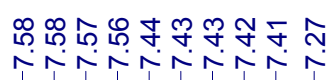

$$
\begin{aligned}
& \begin{array}{l}
1.00 \\
0.95 \\
0.90
\end{array} \\
& \begin{array}{c}
0.90 \\
0.85
\end{array} \\
& \begin{array}{l}
0.85 \\
0.80
\end{array} \\
& \begin{array}{r}
0.75 \\
0.70
\end{array} \\
& \begin{array}{r}
0.70 \text { 害 } \\
0.65
\end{array} \\
& =0.60 \\
& \nsubseteq 0.55 \\
& \stackrel{\oplus}{\stackrel{\bar{\sigma}}{\bar{\sigma}}} 0.50 \text { 奉 } \\
& 0.45 \\
& 0.40 \\
& 0.35 \\
& 0.30 \\
& 0.25 \\
& 0.20 \\
& 0.15 \\
& 0.10 \text { 青 } \\
& \text { 0.05青 } \\
& 0
\end{aligned}
$$

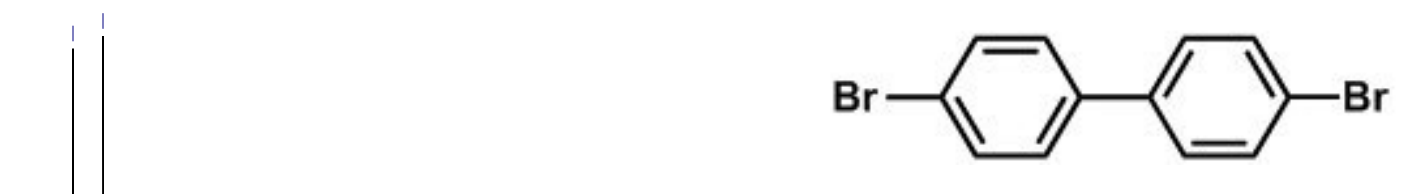

21

4.00 3.96

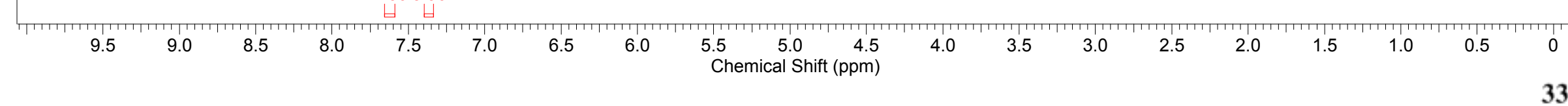




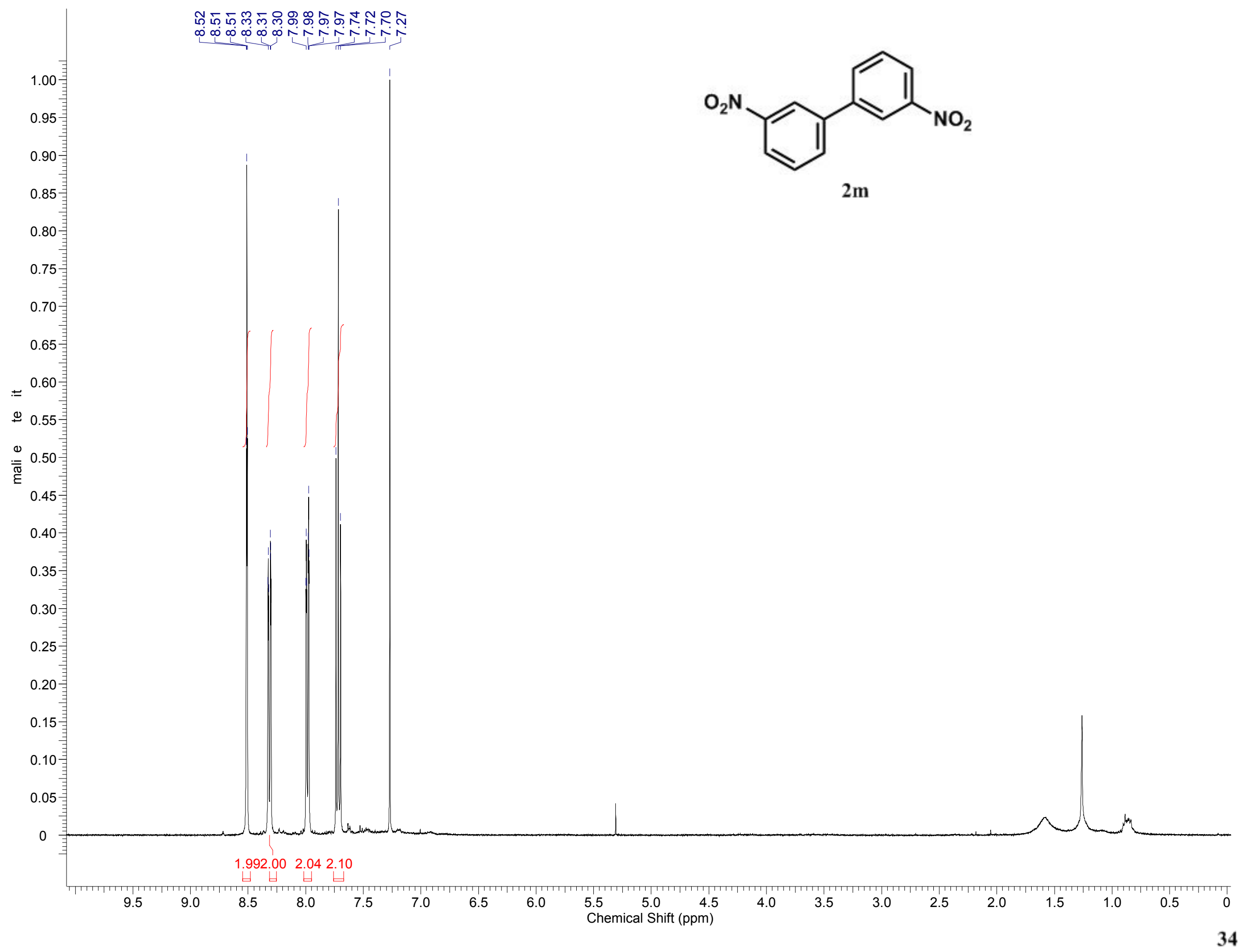




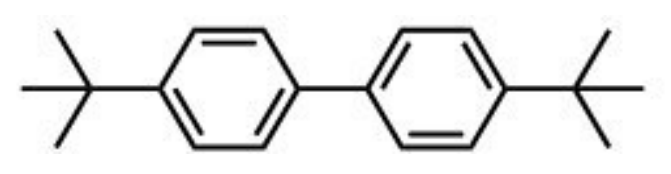

0.10 0.05 当 0 奉

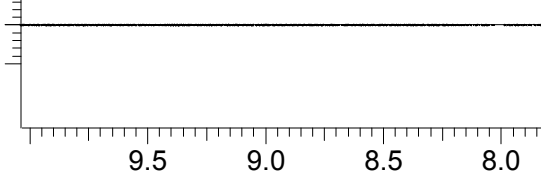




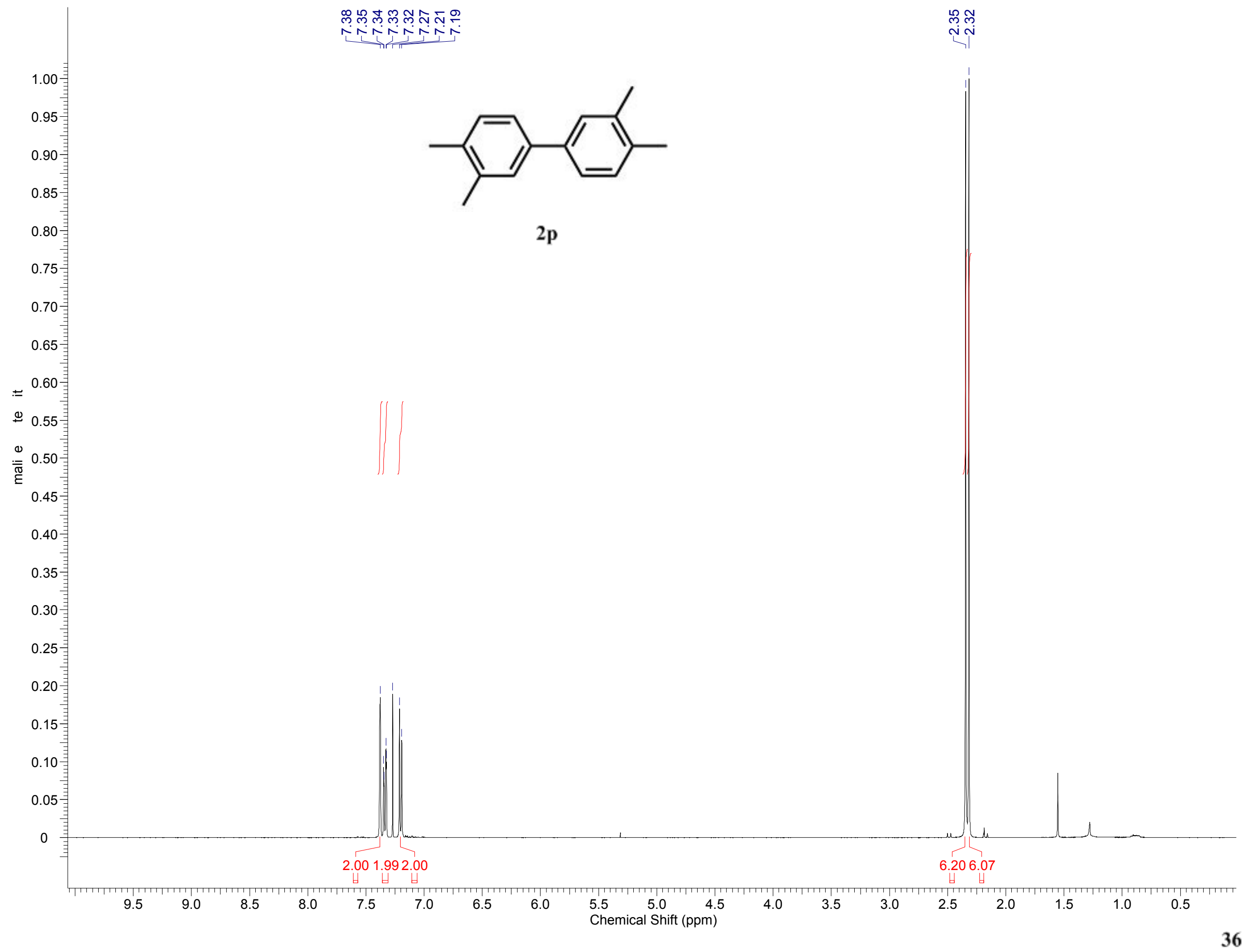




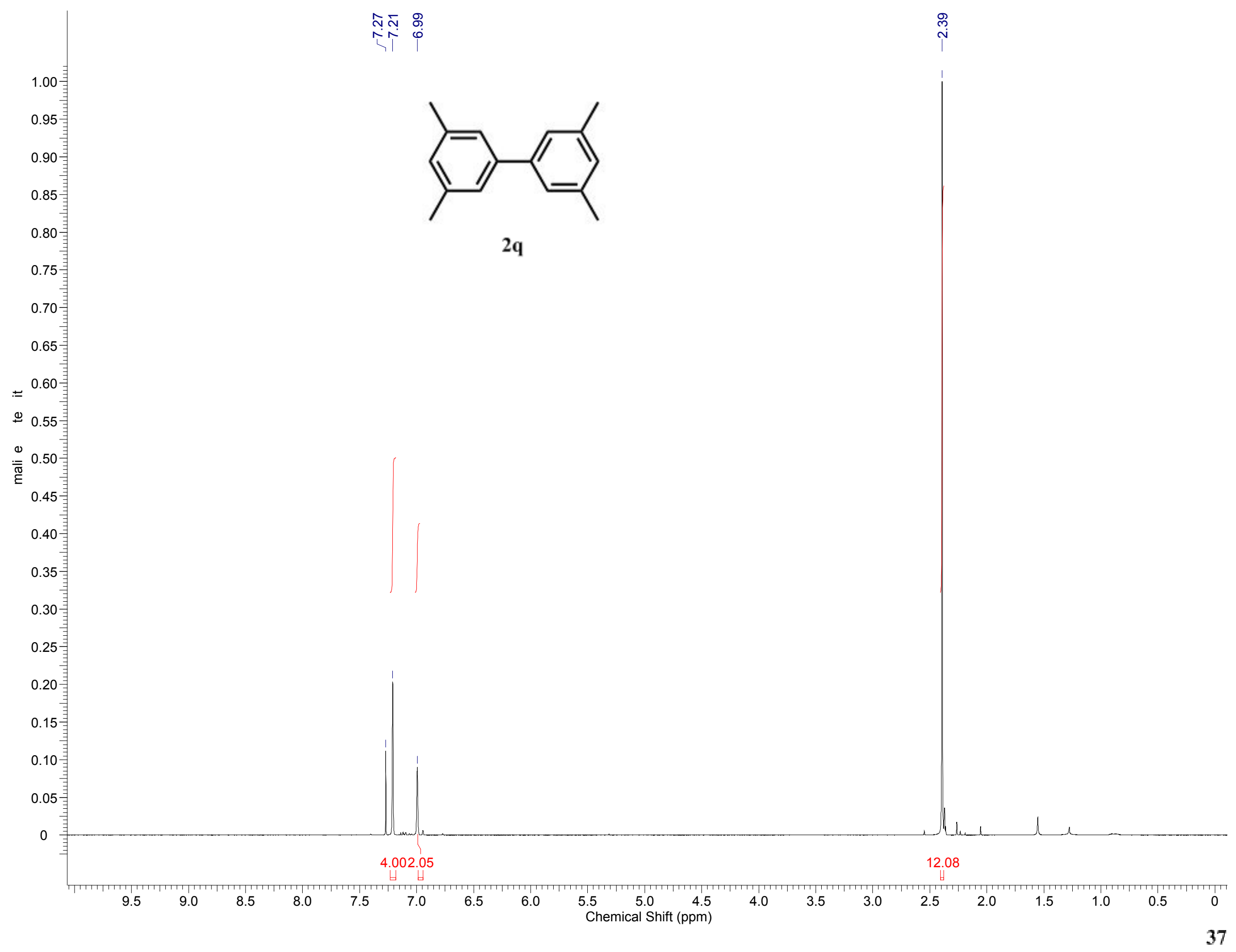




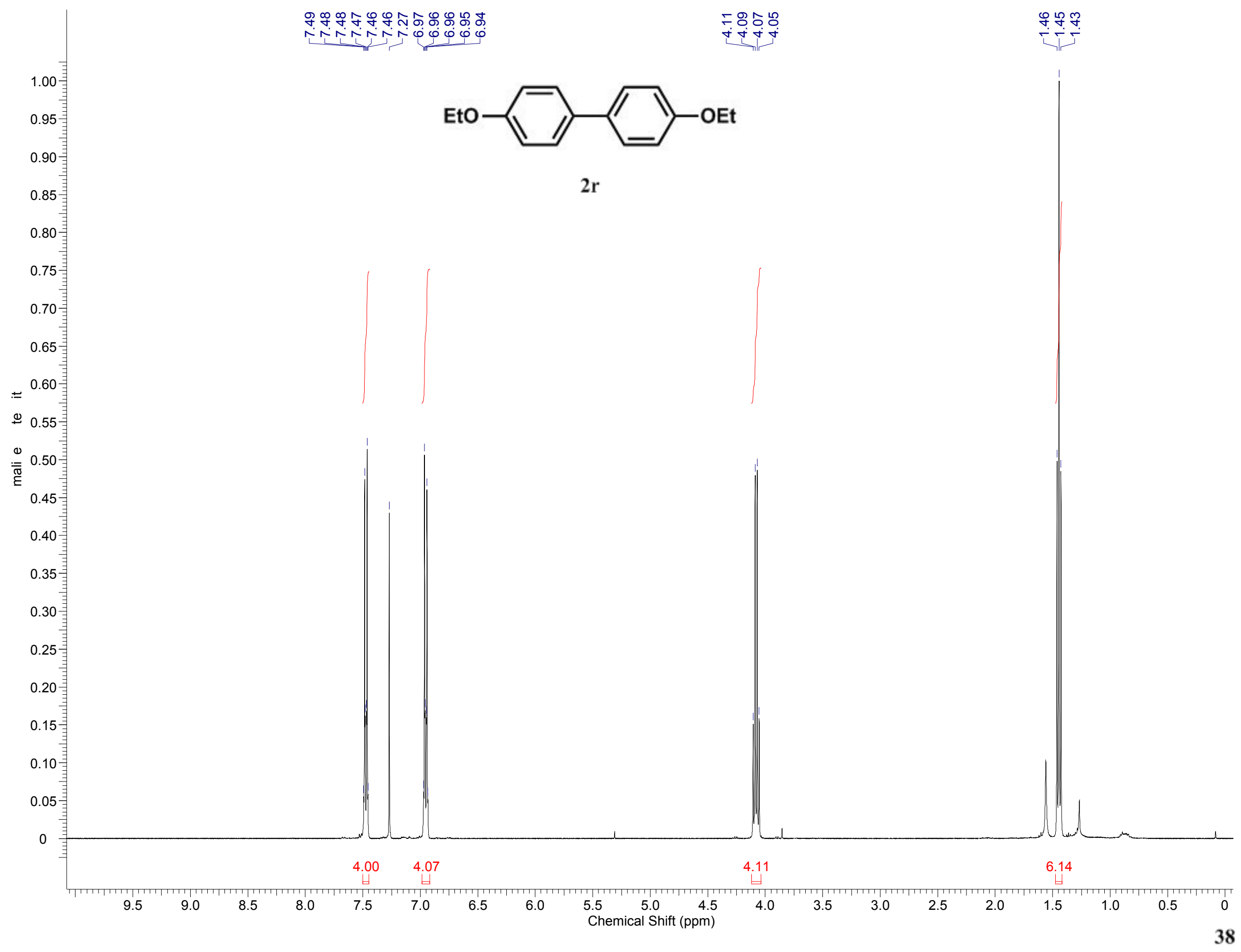




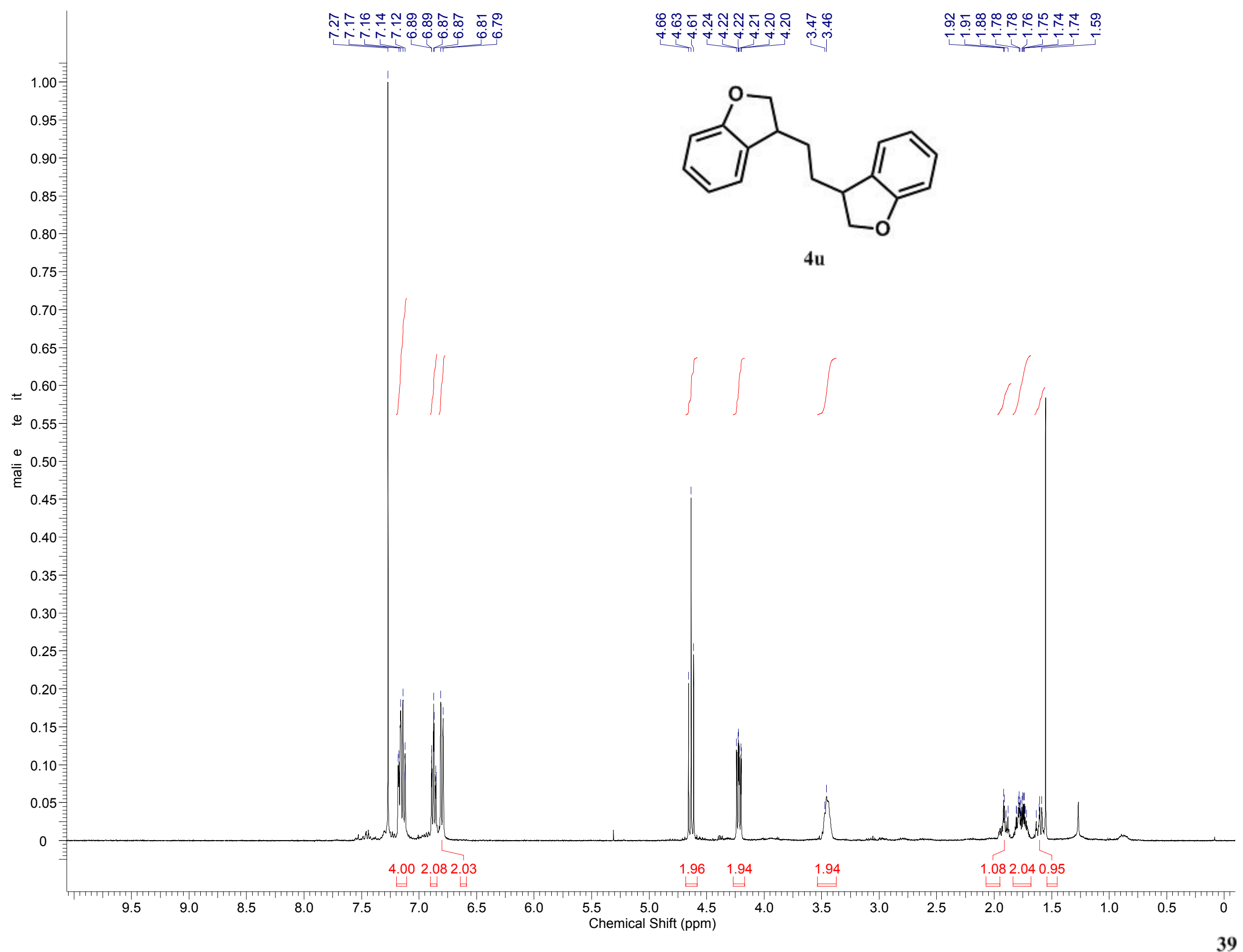




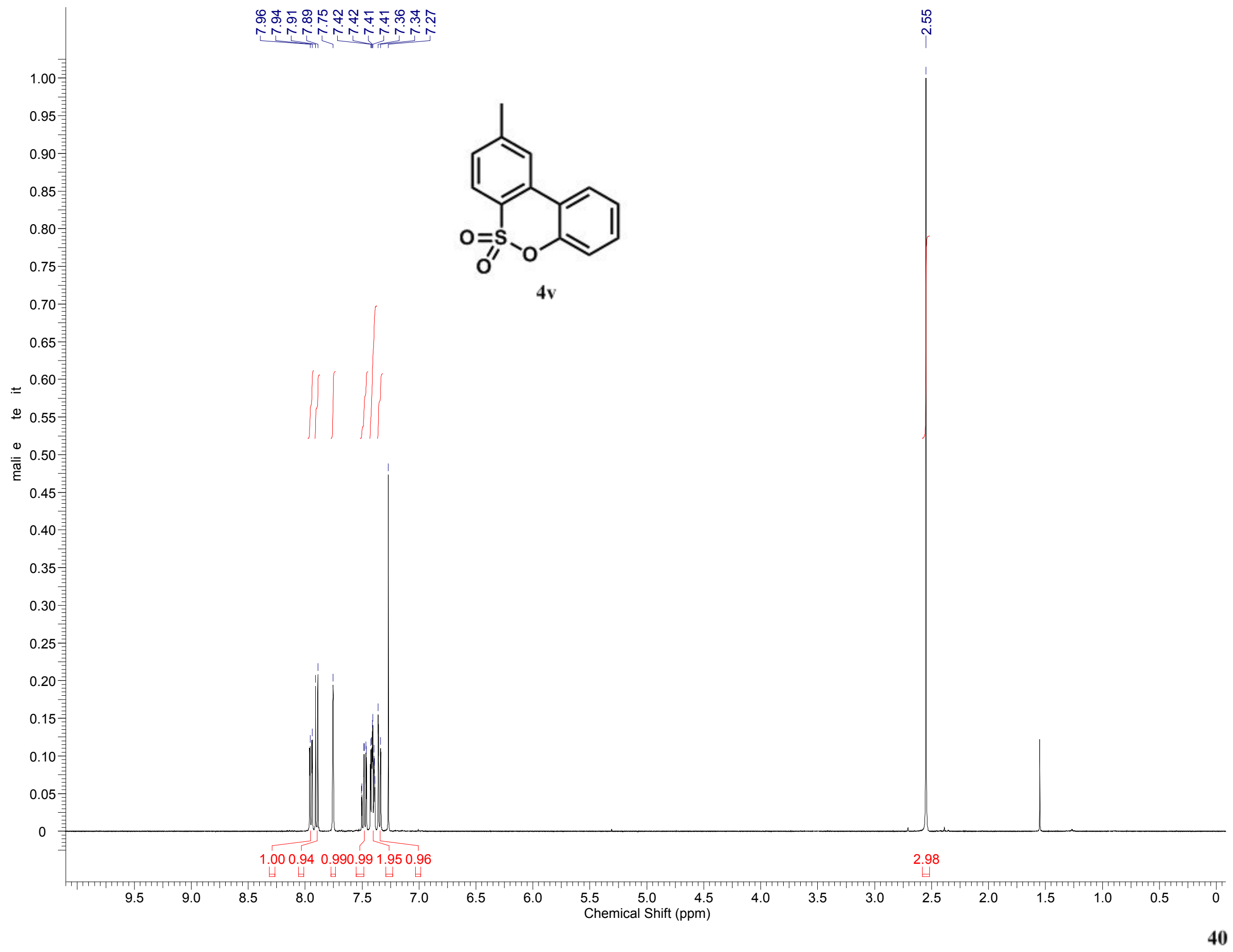




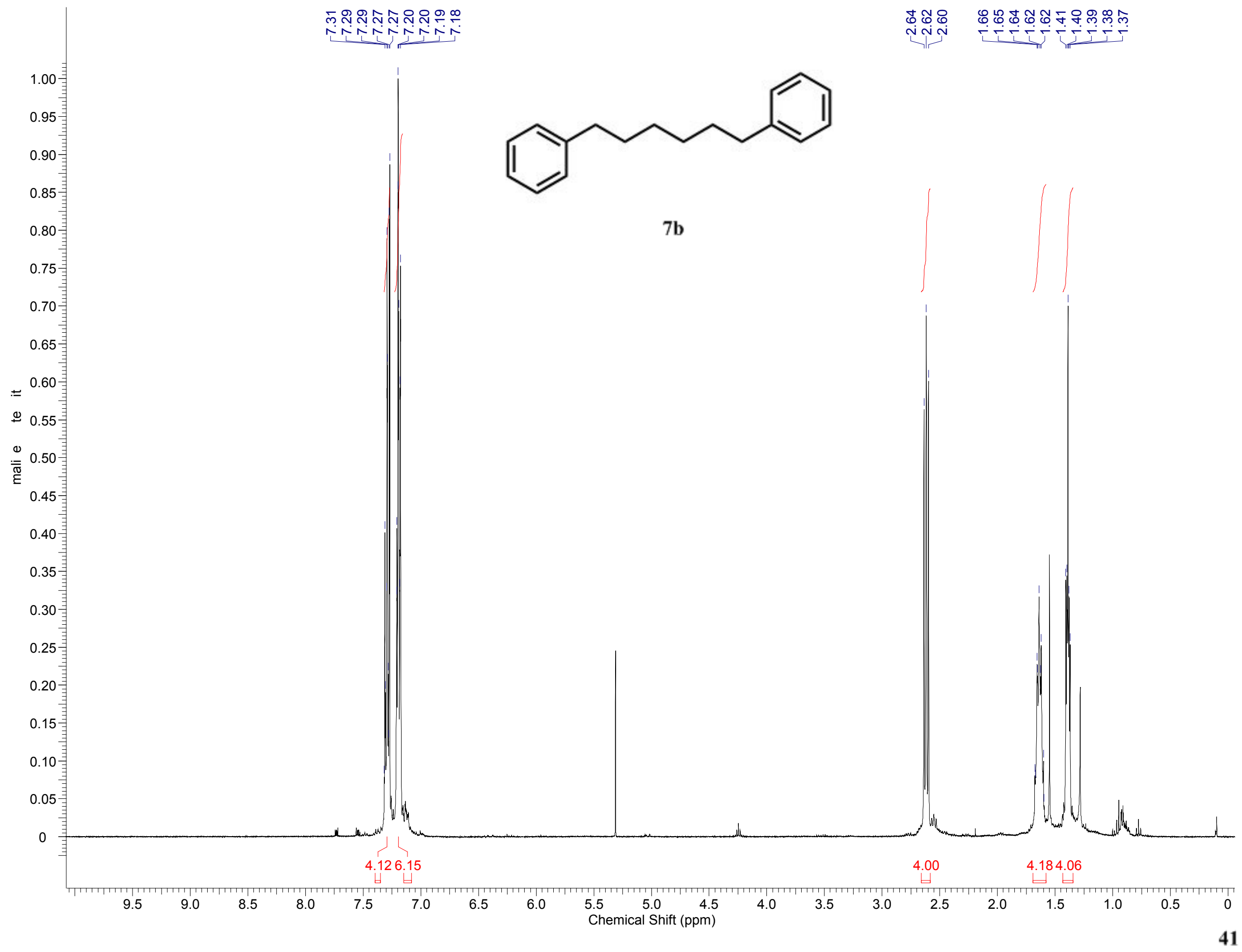




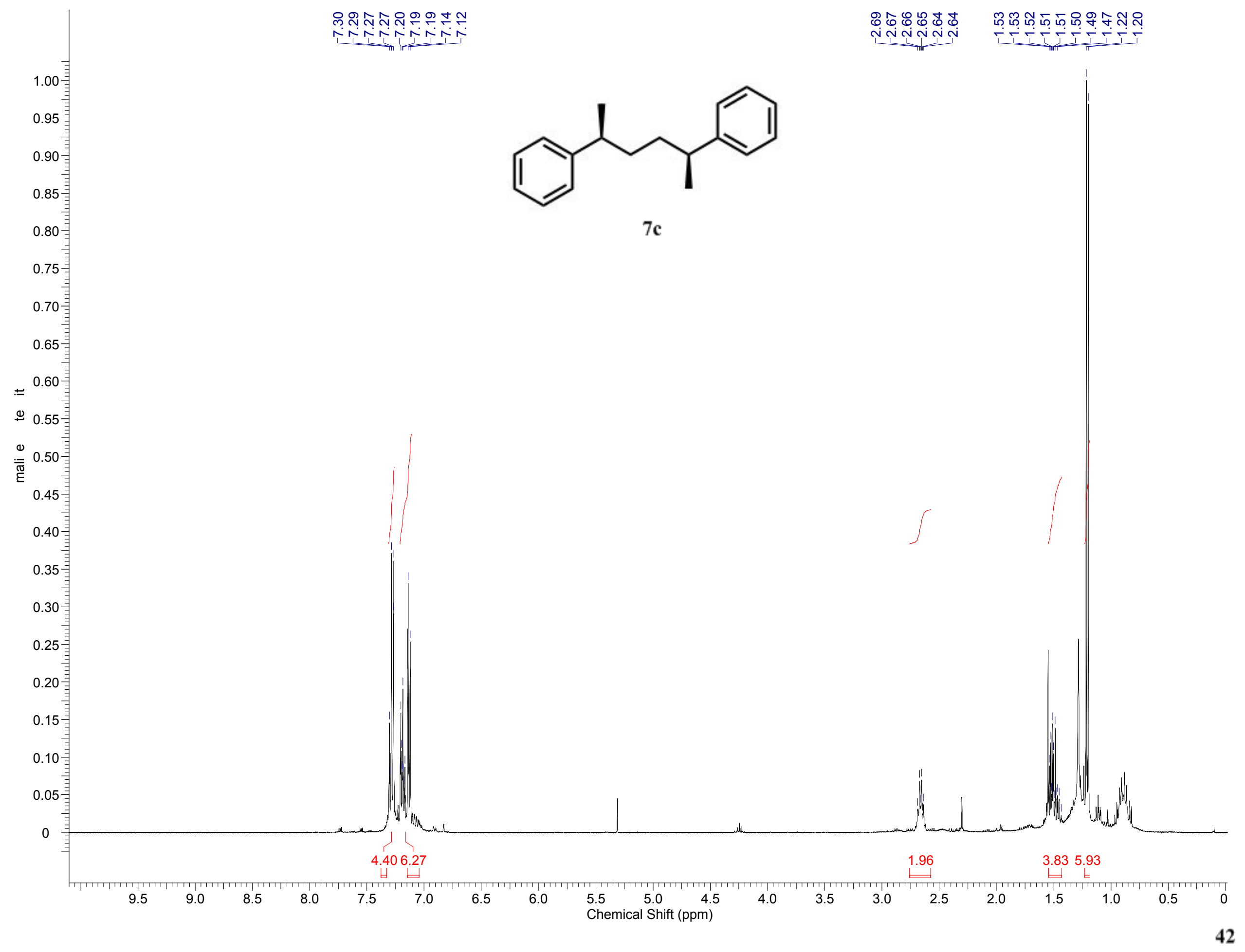




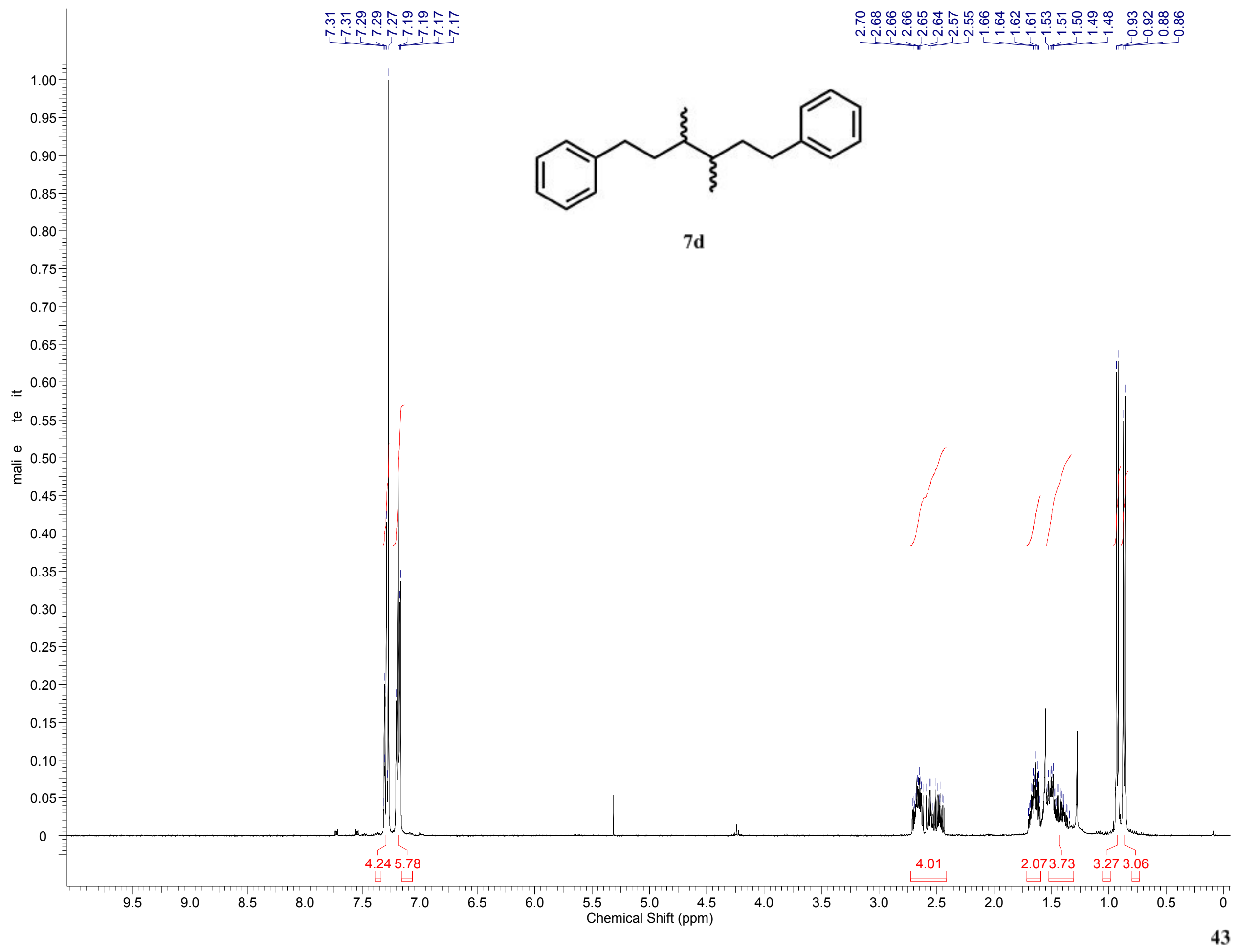


<smiles>c1ccc(CCc2ccccc2)cc1</smiles>

0.75
0.70

0.70
0.65

$=0.60$

Ð 0.55

$\stackrel{0}{\bar{\pi}} 0.50$ 寻

0.45

0.40

0.35

0.30

0.25

0.20

0.15

0.10 慧

0.05 彗

0 青

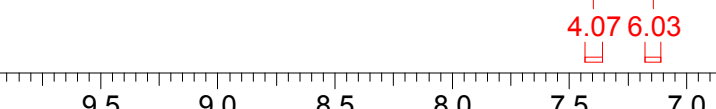




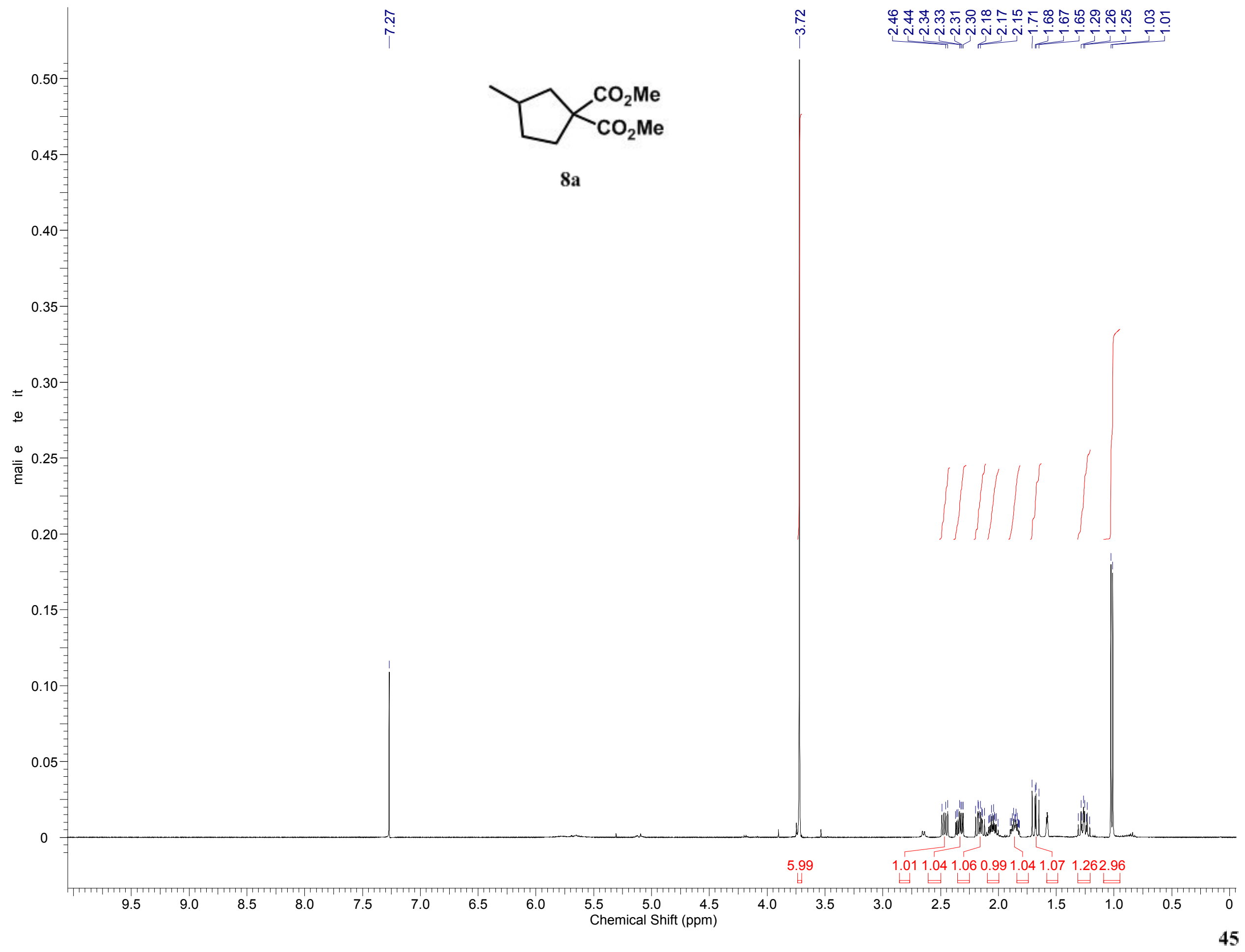

